Prepared in cooperation with the Federal Emergency Management Agency

\title{
Monitoring Storm Tide and Flooding From Hurricane Irma Along the U.S. Virgin Islands, Puerto Rico, and the Southeastern United States, September 2017
}
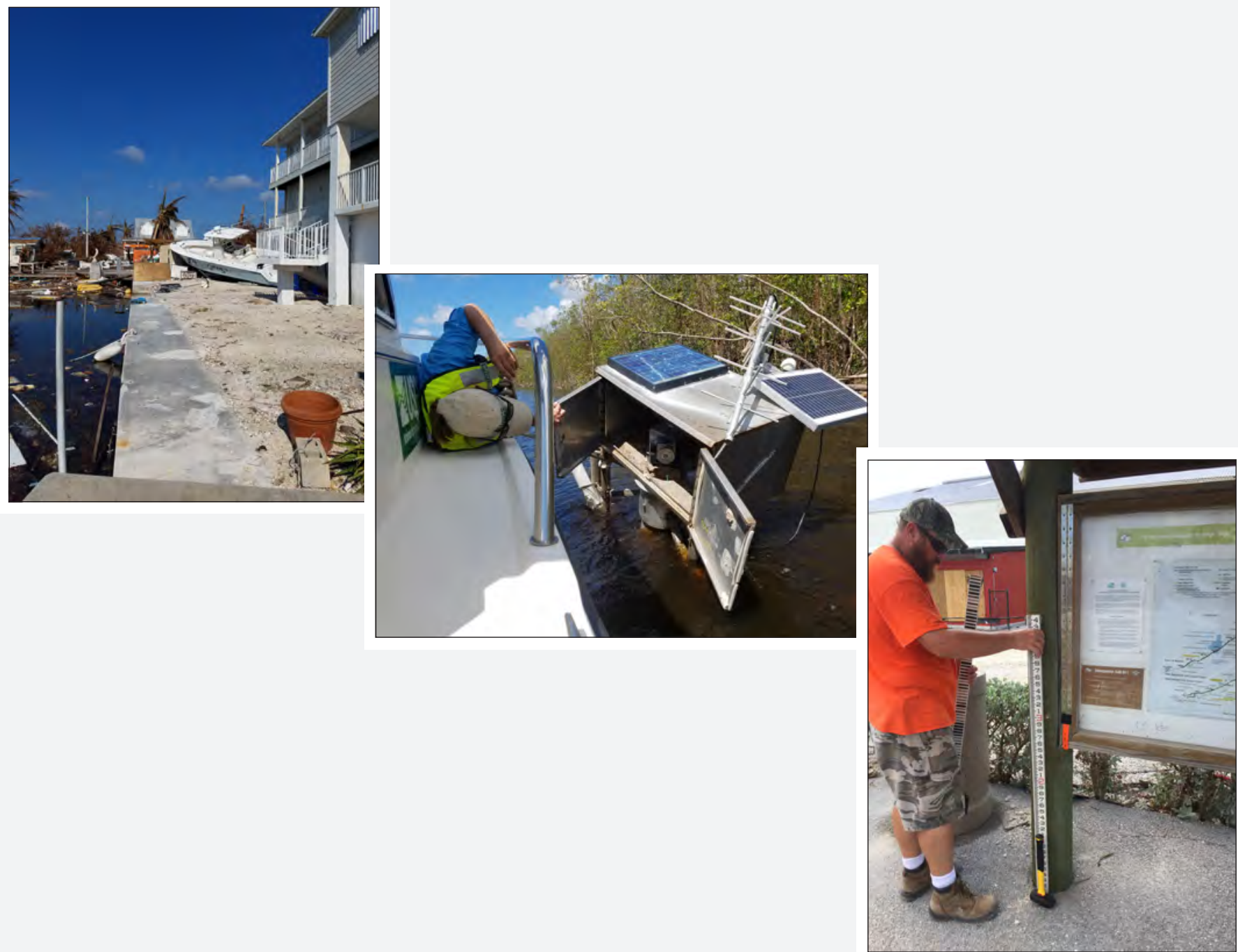

Open-File Report 2019-1013

Version 1.1, July 2019 
Cover. Left: Storm damage in Big Pine Key, Florida. Photograph by Rick Solis, U.S. Geological Survey (USGS). Middle: USGS hydrologic technician Meagan Montgomery inspecting a hurricane-damaged streamgage in Chokoloskee, Florida. Photograph by Robert Clendening, USGS. Right: USGS hydrologic technician David Byers measuring a high-water mark in Big Pine Key, Florida. Photograph by Don Hampton, USGS. 


\section{Monitoring Storm Tide and Flooding From Hurricane Irma Along the U.S. Virgin Islands, Puerto Rico, and the Southeastern United States, September 2017}

By Michael J. Byrne, Sr., and Mark R. Dickman

Prepared in cooperation with the Federal Emergency Management Agency

Open-File Report 2019-1013

Version 1.1, July 2019 


\title{
U.S. Department of the Interior \\ DAVID L. BERNHARDT, Secretary
}

\section{U.S. Geological Survey James F. Reilly II, Director}

\author{
U.S. Geological Survey, Reston, Virginia \\ First release: 2019, online \\ Revised: July 2019 (ver. 1.1), online and in print
}

For more information on the USGS —-the Federal source for science about the Earth, its natural and living resources, natural hazards, and the environment-visit https://www.usgs.gov or call 1-888-ASK-USGS.

For an overview of USGS information products, including maps, imagery, and publications,

visit https://store.usgs.gov.

Any use of trade, firm, or product names is for descriptive purposes only and does not imply endorsement by the U.S. Government.

Although this information product, for the most part, is in the public domain, it also may contain copyrighted materials as noted in the text. Permission to reproduce copyrighted items must be secured from the copyright owner.

Suggested citation:

Byrne, M.J., Sr., and Dickman, M.R., 2019, Monitoring storm tide and flooding from Hurricane Irma along the U.S. Virgin Islands, Puerto Rico, and the Southeastern United States, September 2017 (ver. 1.1, July 2019): U.S. Geological Survey Open-File Report 2019-1013, 35 p., https://doi.org/10.3133/ofr20191013.

ISSN 0196-1497 (print) ISSN 2331-1258 (online) 


\section{Acknowledgments}

This mission would not be possible without the dedication of the U.S. Geological Survey field crew and office personnel as well as the generosity of the private citizens who granted access to their property to survey the hurricane damage. 



\section{Contents}

Acknowledgments …........................................................................................................................

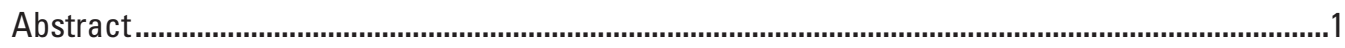

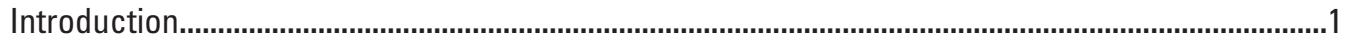

Hurricane Irma Storm-Tide Monitoring....................................................................................

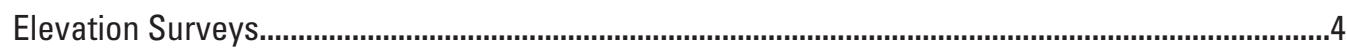

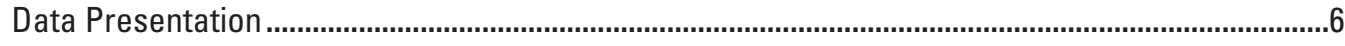

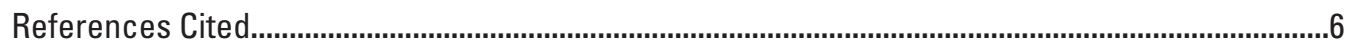

\section{Figures}

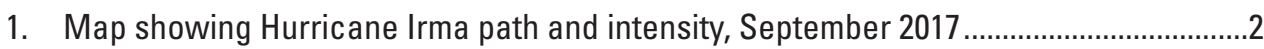

2. Map showing location of water-level sensors for monitoring time, areal extent, and magnitude of storm tide and coastal flooding generated by Hurricane Irma, September 2017

3. Photograph of U.S. Geological Survey hydrologic technician Scott Greenwood installing a water-level sensor in Sebastian Florida...

4. Screenshot of an example hydrograph from Flood Event Viewer displaying storm-tide elevation and barometric pressure data recorded during Hurricane Irma at Goodland, Florida, September 2017

5. Photograph of U.S. Geological Survey rapid-deployment gage collecting real-time water-level and barometric pressure data on Highway U.S. 1 in Islamorada, Florida, September 2017

6. Photograph of U.S. Geological Survey hydrologic technician David Byers measuring high- water mark in Islamorada, Florida...

7. Photograph of a Global Positioning System to survey storm-tide elevation for Hurricane Irma in Chokoloskee, Florida, September 2017.

\section{Tables}

1. Number of sites equipped to monitor Hurricane Irma storm tide, by State and U.S. territory.

2. Hurricane Irma peak storm-tide and wave-height data for $\mathbf{2 4 9}$ temporarily deployed sites, by State and U.S. territory.

3. Hurricane Irma peak storm-tide data recorded at U.S. Geological Survey long-term monitoring sites, by State and U.S. territory...

4. Hurricane Irma peak storm-tide data recorded at State and other Federal agency monitoring sites, by State and U.S. territory

5. Number of sites equipped to monitor Hurricane Irma storm tide, by State and U.S. territory. 


\section{Conversion Factors}

U.S. customary units to International System of Units

\begin{tabular}{lcc}
\hline \multicolumn{1}{c}{ Multiply } & By & To obtain \\
\hline foot $(\mathrm{ft})$ & Length & \\
\hline & 0.3048 & meter $(\mathrm{m})$ \\
\hline mile per hour $(\mathrm{mi} / \mathrm{h})$ & Velocity & \\
\hline
\end{tabular}

\section{Datum}

Vertical coordinate information is referenced to the North American Vertical Datum of 1988 (NAVD 88) or Puerto Rico Vertical Datum of 2002 (PRVD02) unless otherwise noted.

Horizontal coordinate information is referenced to the North American Datum of 1983 (NAD 83).

Elevation, as used in this report, refers to distance above the vertical datum.

\section{Abbreviations}

$\begin{array}{ll}\text { ASCII } & \begin{array}{l}\text { American Standard Code for Information } \\ \text { Interchange }\end{array} \\ \text { GNSS } & \text { Global Navigation Satellite System } \\ \text { HWM } & \text { high-water mark } \\ \text { NetCDF } & \text { Network Common Data Form } \\ \text { RDG } & \text { rapid deployment gage } \\ \text { USGS } & \text { U.S. Geological Survey }\end{array}$




\title{
Monitoring Storm Tide and Flooding From Hurricane Irma Along the U.S. Virgin Islands, Puerto Rico, and the Southeastern United States, September 2017
}

\author{
By Michael J. Byrne, Sr., and Mark R. Dickman
}

\section{Abstract}

Hurricane Irma skirted the northern coasts of the U.S. Virgin Islands and Puerto Rico, with maximum sustained winds of 185 miles per hour (mi/h) on September 6, 2017. The hurricane first made landfall in Florida near Cudjoe Key, in the lower Florida Keys, with maximum sustained winds of $130 \mathrm{mi} / \mathrm{h}$ on September 10, 2017. The hurricane made a second Florida landfall on Marco Island, Florida, with maximum sustained winds of $115 \mathrm{mi} / \mathrm{h}$ on September 10, 2017. The U.S. Geological Survey (USGS), in cooperation with Federal Emergency Management Agency, deployed a temporary monitoring network of water-level and barometric pressure sensors at 249 locations along the Puerto Rico, Florida, Georgia, and South Carolina coasts to record the timing, areal extent, and magnitude of hurricane storm tide and coastal flooding generated by the hurricane. Immediately following the passage of Hurricane Irma, the sensors were retrieved, and the data were disseminated on the USGS Flood Event Viewer (https://stn.wim.usgs.gov/FEV/\#IrmaSeptember2017). The storm-tide peak data values were verified by comparing data from hydrologic recorders and nearby high-water marks (HWMs). Following the hurricane, 508 independent HWM locations were flagged and surveyed relative to the North American Vertical Datum of 1988, National Geodetic Vertical Datum of 1929, or a local datum along the southeastern U.S. coast, and to Puerto Rico Vertical Datum of 2002 in Puerto Rico. Most HWMs were in Florida because of the path of the hurricane. The data from the Hurricane Irma storm-tide network are available on a provisional basis in tab-delimited, American Standard Code for Information Interchange (ASCII) format and Network Common Data Form (NetCDF) format by site for each sensor by using the USGS Flood Event Viewer.

\section{Introduction}

Hurricane Irma skirted the northern coast of the U.S. Virgin Islands and Puerto Rico as a Category 5 hurricane on the Saffir-Simpson scale (National Weather Service, 1972), with maximum sustained winds of 185 miles per hour $(\mathrm{mi} / \mathrm{h})$ on September 6, 2017 (fig. 1). The hurricane first made landfall near Cudjoe Key, in the lower Florida Keys, as a Category 4 hurricane with maximum sustained winds of $130 \mathrm{mi} / \mathrm{h}$ on September 10, 2017 (National Hurricane Center, 2017). The hurricane made a second landfall on Marco Island, Florida, as a Category 3 hurricane with maximum sustained winds of $115 \mathrm{mi} / \mathrm{h}$ on September 10, 2017 (National Hurricane Center, 2017). The hurricane left 23 percent of Puerto Rico (370,000 customers) and 59 percent of Florida (6.1 million customers) without power (U.S. Department of Energy, 2017).

The U.S. Geological Survey (USGS), in cooperation with the Federal Emergency Management Agency, deployed a temporary monitoring network of water-level and barometric pressure sensors at 249 locations along the Puerto Rico, Florida, Georgia, and South Carolina coasts to record the timing, areal extent, and magnitude of hurricane storm tide and coastal flooding generated by Hurricane Irma (fig. 2). Storm tide, as defined by the National Oceanic and Atmospheric Administration (2013), is the water-level rise generated by a combination of storm surge and the astronomical tide during a coastal storm. Storm surge is defined as the water-level rise, caused by a storm, over and above the predicted astronomical tide.

The deployment of water-level and barometric pressure sensors and subsequent high-water mark (HWM) collection were completed as part of a coordinated Federal emergency response as outlined by the Robert T. Stafford Disaster Relief and Emergency Assistance Act (42 USC §5121 et seq.) under a directed mission assignment by the Federal Emergency Management Agency. In addition to the pressure sensors, a total of 508 HWMs were recovered and surveyed following the techniques described in Koenig and others (2016).

During the hurricane, real-time water-level data collected at temporary rapid deployment gages (RDGs, https://water.usgs.gov/hif/programs/projects/rapid deployment_gage_III/), long-term USGS streamgaging stations (https://waterdata.usgs.gov/nwis) with instrumentation used to measure water level and corresponding streamflow, and tide-gage stations were relayed hourly or more frequently, through satellite telemetry, for display on the Flood Event 


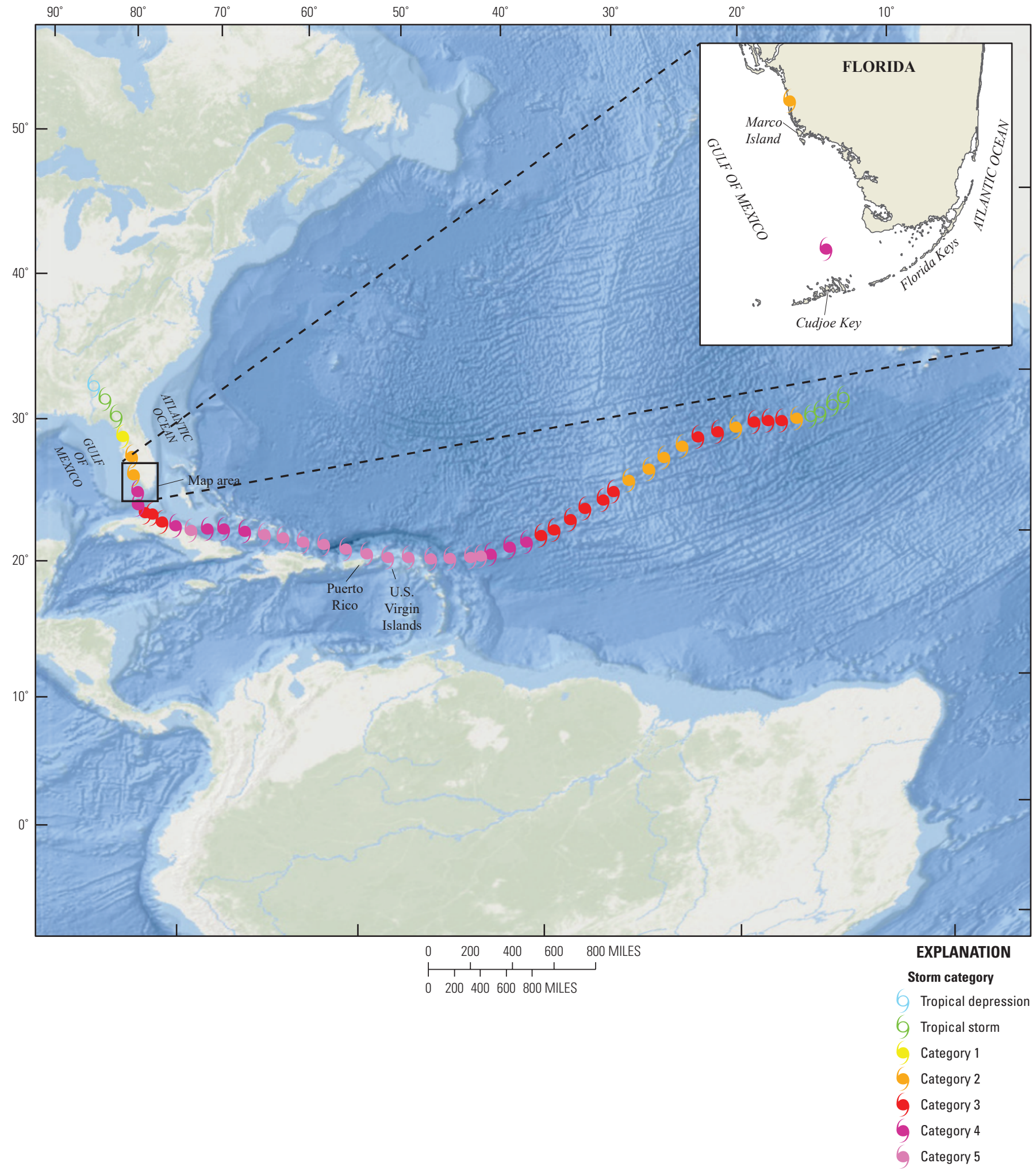

Figure 1. Hurricane Irma path and intensity, September 2017. Map image is the intellectual property of Esri and is used herein under license. Copyright (c) 2017 Esri and its licensors. All rights reserved. 


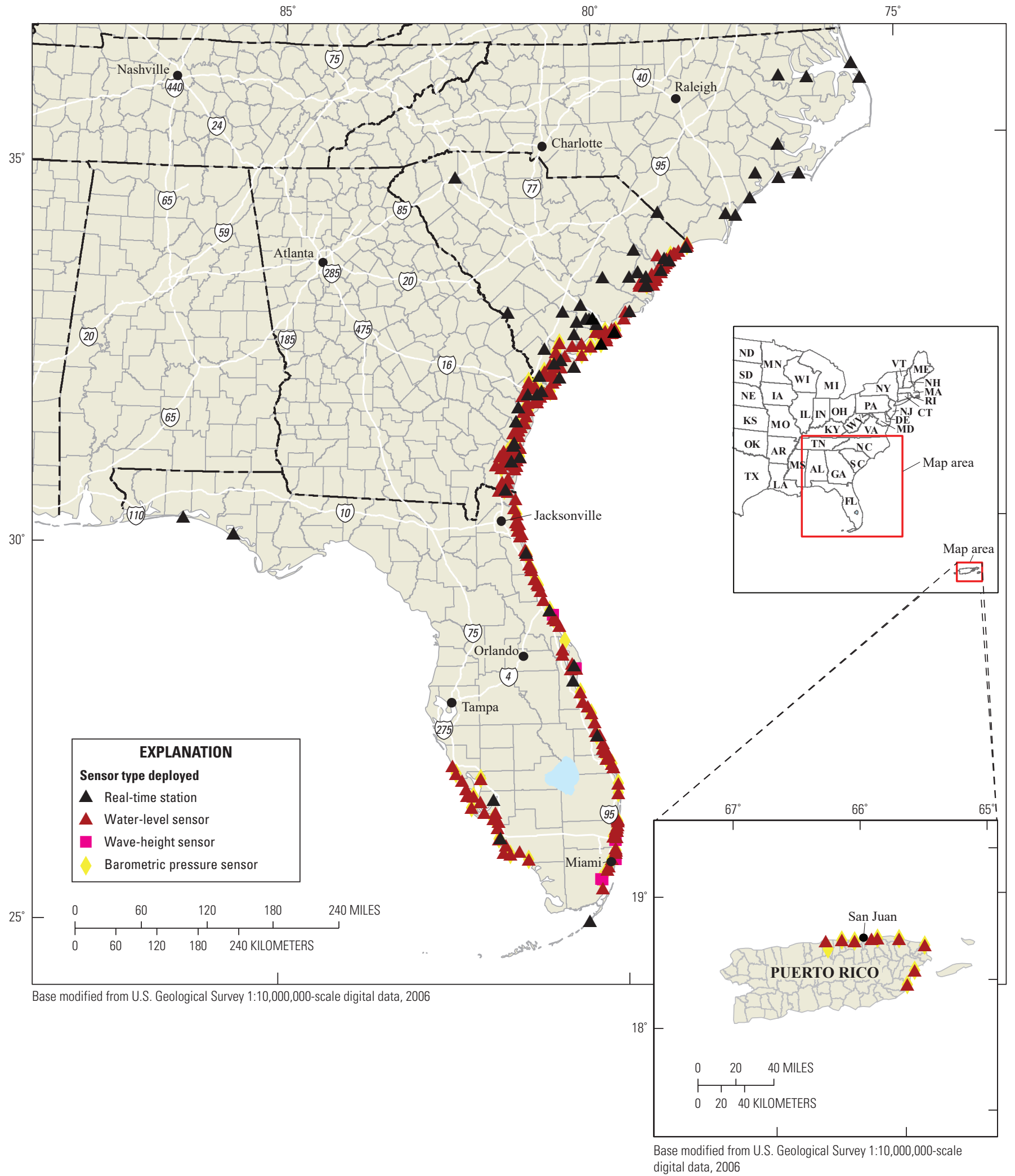

Figure 2. Location of water-level sensors for monitoring time, areal extent, and magnitude of storm tide and coastal flooding generated by Hurricane Irma, September 2017. 
Viewer (https://stn.wim.usgs.gov/FEV/\#IrmaSeptember2017). These real-time data provided emergency managers and responders with critical information for identifying floodaffected areas and accurately directing assistance to affected communities. Data collected during and following this hurricane and others (Frantz and others, 2017) can be used to calibrate and evaluate the performance of storm-tide models used to predict the maximum and incremental water level and flood extent and the site-specific effects of storm tide on natural and anthropogenic features of the environment.

\section{Hurricane Irma Storm-Tide Monitoring}

Water-level sensors were placed at sites selected to augment existing streamgage and tidal-gage networks to ensure adequate monitoring in areas forecast to have had substantive storm tide. A total of 215 water-level sensors, 12 wave-height sensors, 22 RDGs, and 73 barometric pressure sensors were deployed at 249 locations during September 4-10 before hurricane landfall (fig. 2; table 1). Six water-level sensors were lost, and 38 sensors did not record water level because of low water level or equipment malfunction. A typical sensor installation is shown in figure 3.

The sensors recorded water-level elevations in feet above the North American Vertical Datum of 1988 (NAVD 88) or the Puerto Rico Vertical Datum of 2002 (PRVD02). Water-level sensors recorded data at 30 -second intervals and wave-height sensors recorded data at 1 -second or 0.25 -second intervals. The barometric pressure transducers recorded barometric pressure, in pounds per square inch, at 30-second intervals. Water-level elevation and barometric pressure were recorded by the sensors during the hurricane, and an example of the data is shown in figure 4 . The RDG water levels are mean values collected using a radar sensor sampling at 5-second or shorter intervals (Park and others, 2014) and averaged over 6 or 15 minutes. A typical RDG installation is shown in figure 5.

Immediately following Hurricane Irma, the sensors were retrieved, and the data were disseminated on the USGS Flood Event Viewer. Data were collected and processed following protocols established by McGee and others (2006)

Table 1. Number of sites equipped to monitor Hurricane Irma storm tide, by State and U.S. territory.

\begin{tabular}{lrrcc}
\hline & \multicolumn{4}{c}{ Type and number of sensors deployed } \\
\cline { 2 - 5 } $\begin{array}{c}\text { State or } \\
\text { U.S. territory }\end{array}$ & $\begin{array}{c}\text { Water } \\
\text { level }\end{array}$ & $\begin{array}{c}\text { Wave } \\
\text { height }\end{array}$ & $\begin{array}{c}\text { Real-time } \\
\text { rapid } \\
\text { deployment } \\
\text { gages }\end{array}$ & $\begin{array}{c}\text { Barometric } \\
\text { pressure }\end{array}$ \\
\hline Florida & 83 & 12 & 5 & 31 \\
Georgia & 57 & 0 & 8 & 18 \\
South Carolina & 66 & 0 & 9 & 16 \\
Puerto Rico & 9 & 0 & 0 & 8 \\
\hline Total & $\mathbf{2 1 5}$ & $\mathbf{1 2}$ & $\mathbf{2 2}$ & $\mathbf{7 3}$ \\
\hline
\end{tabular}

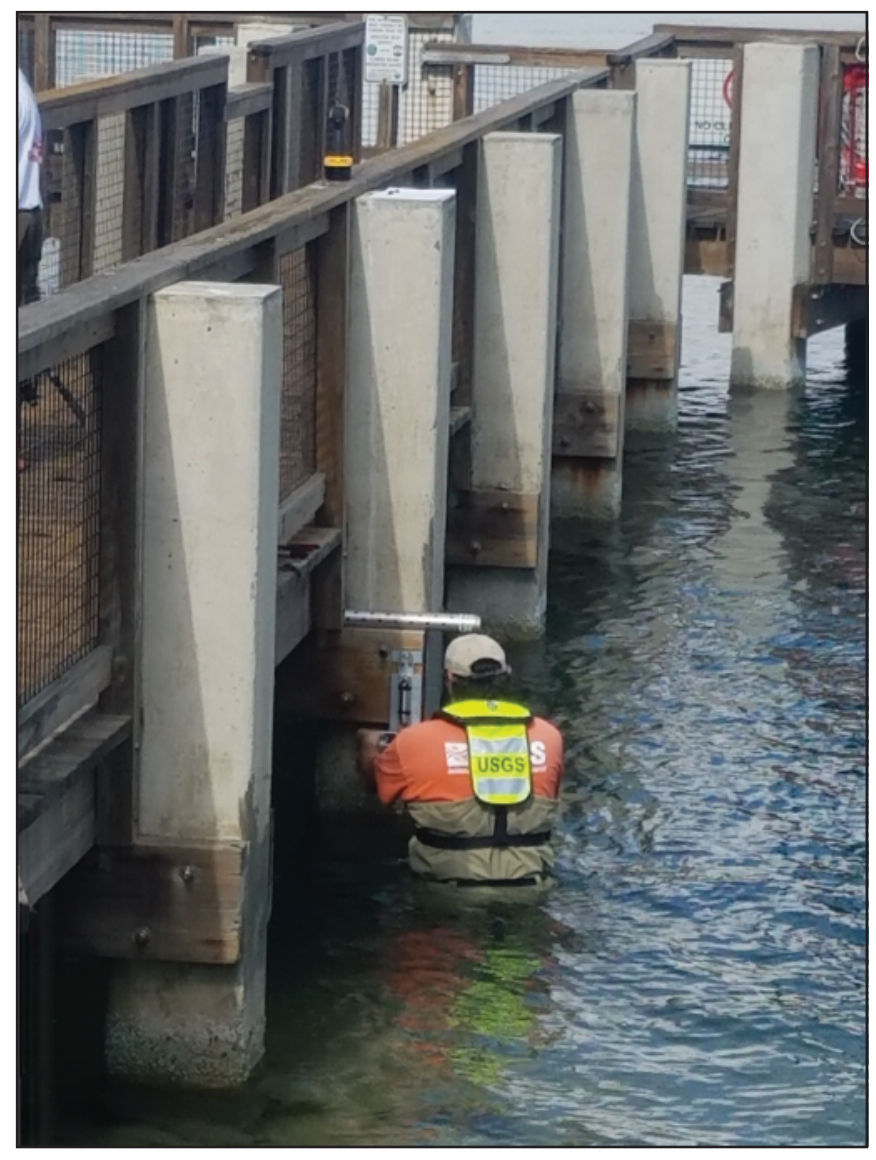

Figure 3. U.S. Geological Survey (USGS) hydrologic technician Scott Greenwood installing a water-level sensor in Sebastian Florida. Photograph by Kagho Asongu, Cherokee Nation and Technology, under contract to the USGS.

and McCallum and others (2012), which included correcting water-level readings for barometric pressure and salinity. The filtered storm-tide peak, as shown in figure 4 was determined using the method described by Frantz and others (2017). The storm-tide peak data values were verified by comparing adjacent hydrologic recorders and HWMs. Following the hurricane, 508 independent HWM locations were flagged and surveyed relative to NAVD 88, National Geodetic Vertical Datum of 1929 (NGVD 29), or a local datum along the Atlantic and Gulf coasts, and PRVD02 in Puerto Rico (fig. 6). Most HWMs were in Florida because of the path of the hurricane.

\section{Elevation Surveys}

National Geodetic Survey benchmarks throughout the study area were surveyed for vertical control. This control was established on permanent objects near the water-level sensors to relate the recorded water-surface elevation to the NAVD 88 or PRVD02 datum. Graduated steel tapes were used to relate the elevations of the reference points to those of the water-level sensors. Survey-grade Global Navigation Satellite System (GNSS) equipment (fig. 7) was used to determine the 


\section{‡USGS \\ science for a changing world}

EXPLANATION

- Unfiltered Water Elevation

- Storm Tide (Lowpass finered) Water Elevation

- - Minimum Recordable Water Elevation

- Barometric Pressure

- Maximum Unfitered Water Elevation

$\Delta$ Maximum Storm Tide Water Elevatio

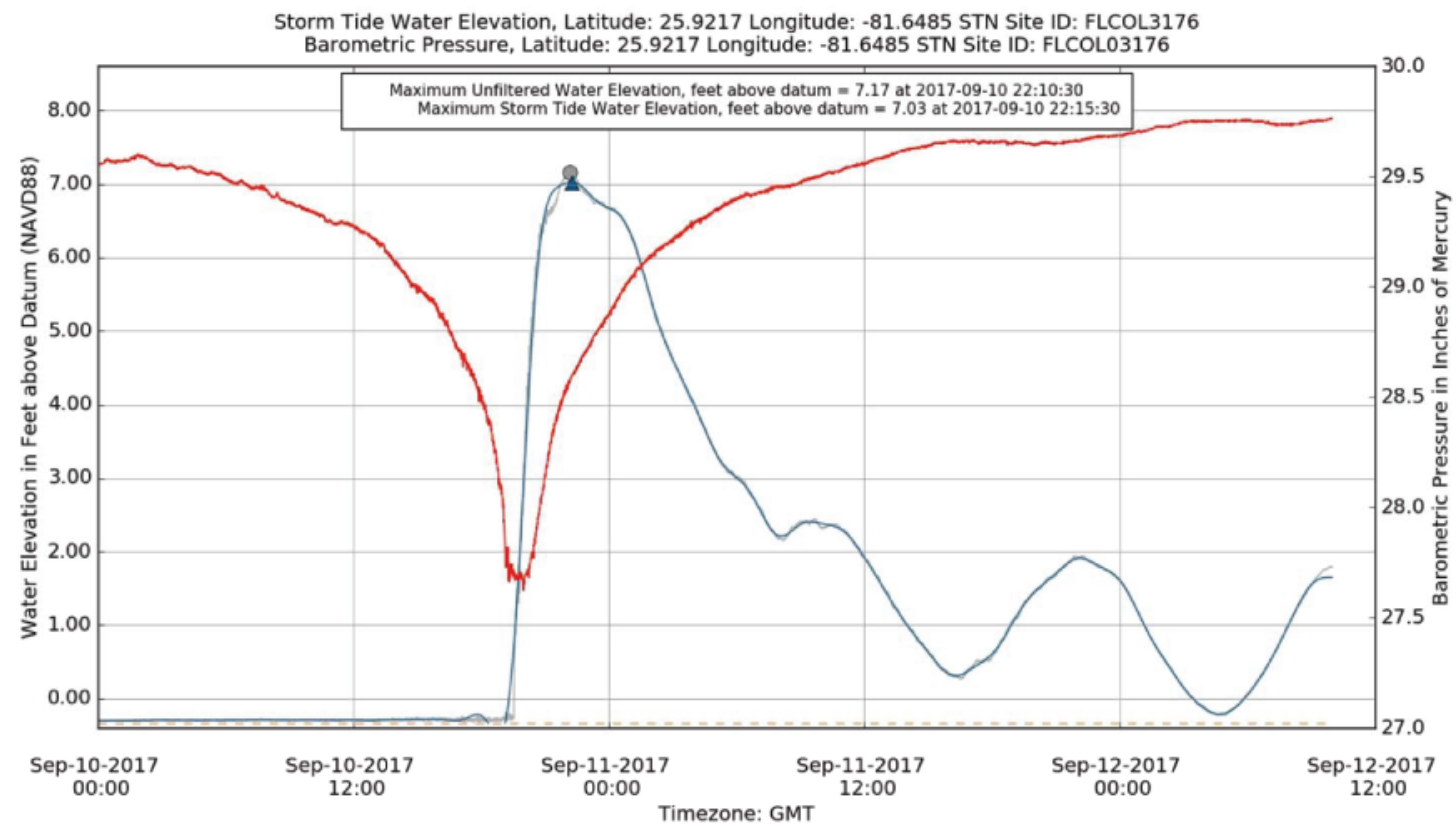

Figure 4. Hydrograph from Flood Event Viewer (https://stn.wim.usgs.gov/FEV/\#IrmaSeptember2017) displaying storm-tide elevation and barometric pressure data recorded during Hurricane Irma at Goodland, Florida, September 2017.

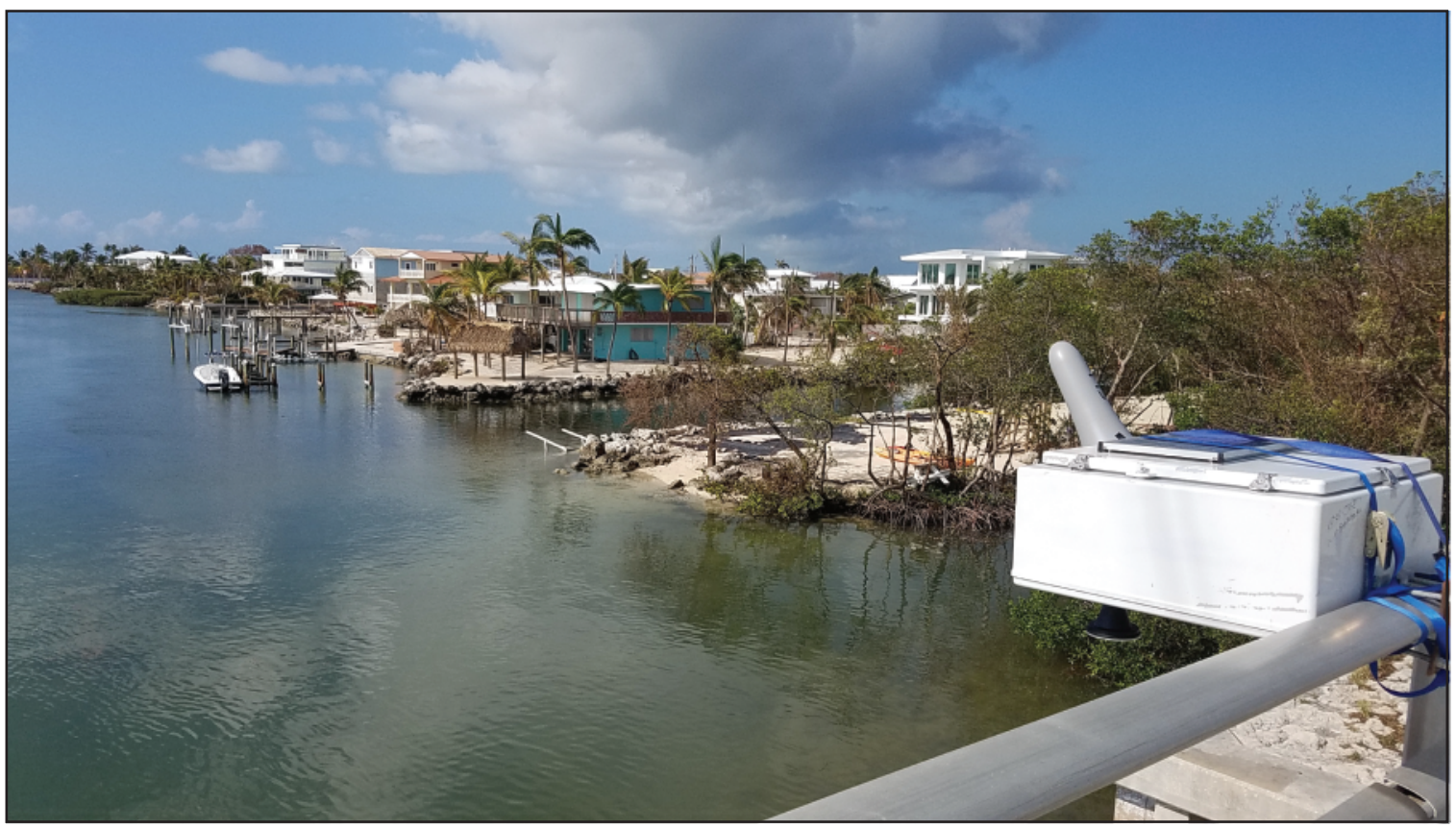

Figure 5. U.S. Geological Survey (USGS) rapid-deployment gage collecting real-time water-level and barometric pressure data on Highway U.S. 1 in Islamorada, Florida, September 2017. Photograph by Sean Raabe, USGS. 


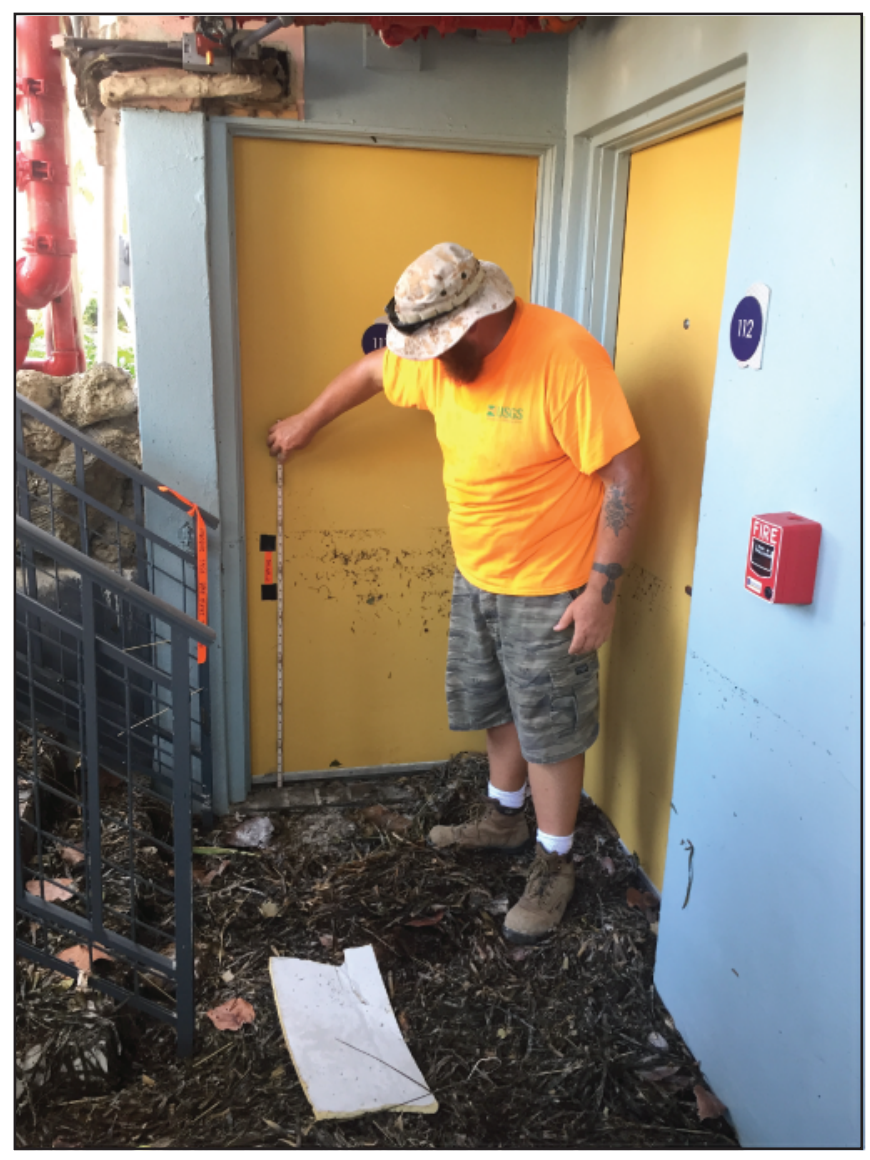

Figure 6. U.S. Geological Survey (USGS) hydrologic technician David Byers measuring high- water mark in Islamorada, Florida. Photograph by Don Hampton, USGS.

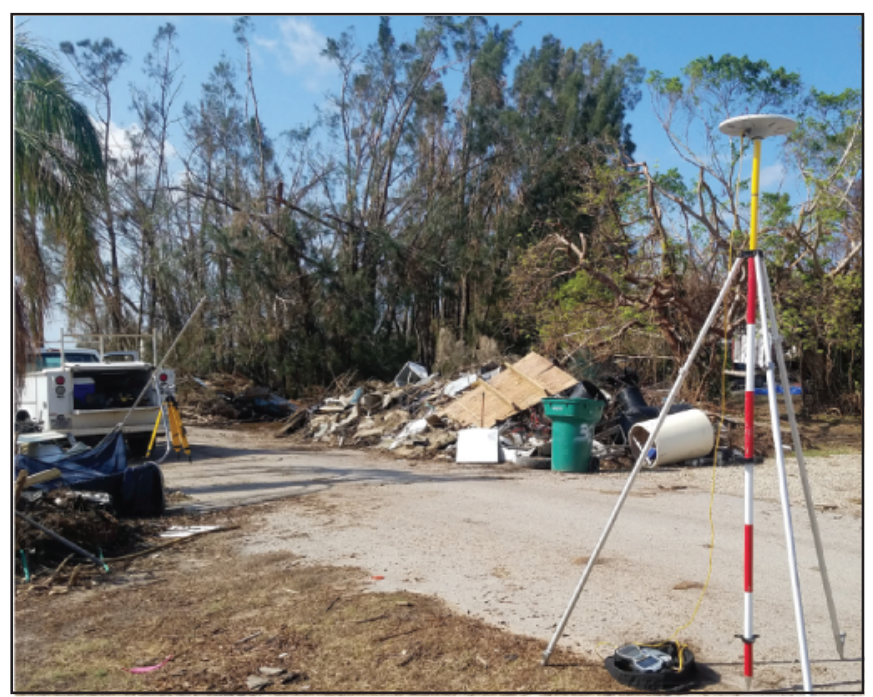

Figure 7. Global Positioning System to survey storm-tide elevation for Hurricane Irma in Chokoloskee, Florida, September 2017. Photograph by Ryan Hollins, U.S. Geological Survey. elevation above NAVD 88 or PRVD02 of the reference points and HWMs, in accordance with USGS technical guidance (Rydlund and Densmore, 2012). All GNSS elevations in this report were derived using the GEOID12B model (National Geodetic Survey, 2017).

\section{Data Presentation}

The data from the Hurricane Irma storm-tide network are available on a provisional basis in tab-delimited, American Standard Code for Information Interchange (ASCII) format and Network Common Data Form (NetCDF) format by site for each sensor by using the USGS Flood Event Viewer. Digital photographs for selected locations are also available on the viewer. Data available for each sensor include location, date, time, water level, and barometric pressure. Data available for HWMs include location, description and quality of the mark, and elevation.

The peak storm tide was recorded at a total of 205 water-level sensors (table 2, at the back of the report). Sites are categorized as storm tide or wave height depending on the data-collection interval and proximity to the ocean. For GNSS established elevations, the survey uncertainty of the recorded peak storm-tide is reported (table 2). The survey uncertainty was calculated using the methods described in Rydlund and Densmore (2012). The recorded peak storm-tide at the long-term USGS monitoring stations along the coasts of Puerto Rico, Florida, Georgia, and South Carolina is provided in table 3 . In addition to the storm-tide data collected by the USGS, peak storm-tide elevations also were compiled for real-time monitoring stations operated by State and other Federal agencies (table 4). The North Carolina Division of Emergency Management provided data for three of their monitoring stations (David Herlong, North Carolina Division of Emergency Management, written commun., February 2017). Peak storm-tide data also were compiled for National Oceanic and Atmospheric Administration tide gages (https://tidesandcurrents.noaa.gov/stations. html?type=Water+Levels), which have instrumentation to measure coastal water level located in each State (National Oceanic and Atmospheric Administration, 2017) (table 4). All HWM data collected by the USGS immediately after Hurricane Irma are listed in table 5.

\section{References Cited}

Frantz, E.R., Byrne, M.J., Caldwell, A.W., and Harden, S.L., 2017, Monitoring storm tide and flooding from Hurricane Matthew along the Atlantic Coast of the United States, October 2016: U.S. Geological Survey Open-File Report 2017-1122, 37 p., accessed November 8, 2018, at https://doi.org/10.3133/ofr20171122. 
Koenig, T.A., Bruce, J.L., O’Connor, J.E., McGee, B.D., Holmes, R.R., Jr., Hollins, R., Forbes, B.T., Kohn, M.S., Schellekens, M.F., Martin, Z.W., and Peppler, M.C., 2016, Identifying and preserving high-water mark data: U.S. Geological Survey Techniques and Methods, book 3, chap. A24, 47 p., accessed November 8, 2018, at https://doi.org/10.3133/tm3A24.

McCallum, B.E., Painter, J.A., and Frantz, E.R., 2012, Monitoring storm tide and flooding from Hurricane Irene along the Atlantic Coast of the United States, August 2011: U.S. Geological Survey Open-File Report 2012-1022. [Also available at https://pubs.er.usgs.gov/publication/ ofr20121022.]

McGee, B.D., Goree, B.B., Tollett, R.W., Woodward, B.K., and Kress, W.H., 2006, Hurricane Rita surge data, southwestern Louisiana and southeastern Texas, September to November 2005: U.S. Geological Survey Data Series 220. [Also available at https://pubs.er.usgs.gov/publication/ ds220.]

National Geodetic Survey, 2017, Notice-NGS update, May 16, 2017, Technical details for GEOID12/12A/12B: National Geodetic Survey Geoid web page, accessed February 27, 2018, at https://www.ngs.noaa.gov/GEOID/ GEOID12B/GEOID12B_TD.shtml.

National Oceanic and Atmospheric Administration, 2013, Storm QuickLook: National Oceanic and Atmospheric Administration web page, accessed February 27, 2018, at https://tidesandcurrents.noaa.gov/quicklook.html.
National Oceanic and Atmospheric Administration, 2017, Tides \& Currents: National Oceanic and Atmospheric Administration web page, accessed February 27, 2018, at https://tidesandcurrents.noaa.gov/stations.html?type $=$ Water + Levels.

National Weather Service, 1972, The Saffir-Simpson Hurricane Wind Scale [Updated February 1, 2012]: National Hurricane Center web page, accessed February 27, 2018, at http://www.nhc.noaa.gov/aboutsshws.php.

Park, J., Heitsenrether, R., and Sweet, W., 2014, Water level and wave height estimates at NOAA tide stations from acoustic and microwave sensors: Journal of Atmospheric and Oceanic Technology, v. 31, accessed November 8, 2018, at https://journals.ametsoc.org/doi/abs/10.1175/JTECHD-14-00021.1.

Rydlund, P.H., Jr., and Densmore, B.K., 2012, Methods of practice and guidelines for using survey-grade Global Navigation Satellite Systems (GNSS) to establish vertical datum in the United States Geological Survey: U.S. Geological Survey Techniques and Methods, book 11, chap. D1, 102 p., with appendixes. [Also available online at https://pubs. er.usgs.gov/publication/tm11D1.]

U.S. Department of Energy, 2017, Hurricane Irma \& Hurricane Harvey: U.S. Department of Energy Event Summary, Report No. 26, accessed at March 1, 2018, at https://energy. gov/sites/prod/files/2017/10/f37/hurricanes-irma-and-harvey-event-summary-26.pdf. 

Tables 2-5 
Table 2. Hurricane Irma peak storm-tide and wave-height data for 249 temporarily deployed sites, by State and U.S. territory.

[Dates shown as month, day, year, referenced to Coordinated Universal Time (UTC). ft, foot; NAVD 88, North American Vertical Datum of 1988; RDG, rapid deployment gage; State or U.S. territory: Fla., Florida; Ga., Georgia; N.C., North Carolina; P.R. Puerto Rico; S.C., South Carolina. \pm , plus or minus; na, not available]

\begin{tabular}{|c|c|c|c|c|c|c|c|c|c|}
\hline $\begin{array}{c}\text { Site } \\
\text { identification }\end{array}$ & $\begin{array}{c}\text { State } \\
\text { or U.S. } \\
\text { territory }\end{array}$ & County & $\begin{array}{l}\text { Latitude } \\
\text { Decimal }\end{array}$ & degrees & $\begin{array}{c}\text { Sensor } \\
\text { deployment } \\
\text { type }\end{array}$ & $\begin{array}{l}\text { Type of data } \\
\text { collected }\end{array}$ & $\begin{array}{c}\text { Peak } \\
\text { storm-tide } \\
\text { elevation } \\
\text { (ft above } \\
\text { NAVD 88) }\end{array}$ & $\begin{array}{l}\text { Peak storm tide } \\
\text { elevation date }\end{array}$ & $\begin{array}{l}\text { Surveyed } \\
\text { sensor } \\
\text { elevation } \\
\text { uncertainty } \\
( \pm \mathrm{ft})\end{array}$ \\
\hline FLBAY03331 & Fla. & Bay & 30.188 & -85.742 & Real-time RDG & Storm tide & None & No surge recorded & 0.16 \\
\hline FLBRE03152 & Fla. & Brevard & 28.358 & -80.679 & Water level & Storm tide & 4.87 & 09/11/2017 & 0.20 \\
\hline FLBRE03161 & Fla. & Brevard & 28.553 & -80.795 & Water level & Storm tide & 1.67 & 09/11/2017 & 0.01 \\
\hline FLBRE03165 & Fla. & Brevard & 28.624 & -80.796 & Water level & None & None & Lost equipment & 0.14 \\
\hline FLBRE03167 & Fla. & Brevard & 28.368 & -80.602 & Water level & None & None & No surge recorded & 0.01 \\
\hline FLBRE03169 & Fla. & Brevard & 28.436 & -80.660 & Water level & None & None & No surge recorded & 0.14 \\
\hline FLBRO03495 & Fla. & Broward & 26.058 & -80.111 & Wave height & None & None & No surge recorded & 0.15 \\
\hline FLBRO03496 & Fla. & Broward & 26.059 & -80.128 & Water level & Storm tide & 2.54 & 09/10/2017 & 0.00 \\
\hline FLBRO03521 & Fla. & Broward & 26.317 & -80.075 & Water level & None & None & Lost equipment & na \\
\hline FLBRO03524 & Fla. & Broward & 26.261 & -80.084 & Water level & None & None & Lost equipment & 0.15 \\
\hline FLBRO03525 & Fla. & Broward & 26.222 & -80.104 & Water level & Storm tide & 3.41 & 09/10/2017 & 0.01 \\
\hline FLBRO03527 & Fla. & Broward & 26.189 & -80.094 & Water level & Storm tide & 4.04 & 09/10/2017 & 0.15 \\
\hline FLBRO03530 & Fla. & Broward & 26.114 & -80.106 & Water level & None & None & No surge recorded & 0.13 \\
\hline FLBRO20853 & Fla. & Broward & 26.235 & -80.088 & Water level & Storm tide & 3.72 & 09/10/2017 & 0.15 \\
\hline FLBRO20894 & Fla. & Broward & 26.191 & -80.107 & Water level & Storm tide & 3.28 & $09 / 10 / 2017$ & 0.15 \\
\hline FLCOL03176 & Fla. & Collier & 25.922 & -81.648 & Water level & Storm tide & 7.17 & 09/10/2017 & 0.02 \\
\hline FLCOL03237 & Fla. & Collier & 25.845 & -81.387 & Water level & Storm tide & 8.92 & 09/10/2017 & 0.02 \\
\hline FLCOL03294 & Fla. & Collier & 26.278 & -81.825 & Water level & Storm tide & 3.91 & $09 / 11 / 2017$ & 0.01 \\
\hline FLCOL03296 & Fla. & Collier & 26.132 & -81.806 & Water level & Storm tide & 6.25 & 09/10/2017 & 0.07 \\
\hline FLCOL03297 & Fla. & Collier & 26.142 & -81.790 & Real-time RDG & Storm tide & 4.13 & 09/10/2017 & 0.01 \\
\hline FLDUV03108 & Fla. & Duval & 30.511 & -81.461 & Water level & Storm tide & 6.76 & 09/11/2017 & 0.01 \\
\hline FLDUV03110 & Fla. & Duval & 30.421 & -81.421 & Water level & Storm tide & 6.86 & $09 / 11 / 2017$ & 0.13 \\
\hline FLDUV03114 & Fla. & Duval & 30.288 & -81.421 & Water level & Storm tide & 5.16 & 09/11/2017 & 0.10 \\
\hline FLDUV17794 & Fla. & Duval & 30.324 & -81.435 & Water level & Storm tide & 5.39 & $09 / 11 / 2017$ & 0.00 \\
\hline FLDUV21045 & Fla. & Duval & 30.294 & -81.388 & Water level & Storm tide & 9.9 & $09 / 11 / 2017$ & 0.01 \\
\hline FLFLA03131 & Fla. & Flagler & 29.559 & -81.176 & Water level & Storm tide & 4.98 & $09 / 11 / 2017$ & 0.09 \\
\hline FLFLA03133 & Fla. & Flagler & 29.507 & -81.140 & Water level & Storm tide & None & Lost equipment & 0.05 \\
\hline FLFLA03134 & Fla. & Flagler & 29.480 & -81.126 & Water level & Storm tide & None & No surge recorded & 0.05 \\
\hline
\end{tabular}


Table 2

Table 2. Hurricane Irma peak storm-tide and wave-height data for 249 temporarily deployed sites, by State and U.S. territory.-Continued

[Dates shown as month, day, year, referenced to Coordinated Universal Time (UTC). ft, foot; NAVD 88, North American Vertical Datum of 1988; RDG, rapid deployment gage; State or U.S. territory: Fla., Florida; Ga., Georgia; N.C., North Carolina; P.R. Puerto Rico; S.C., South Carolina. \pm , plus or minus; na, not available]

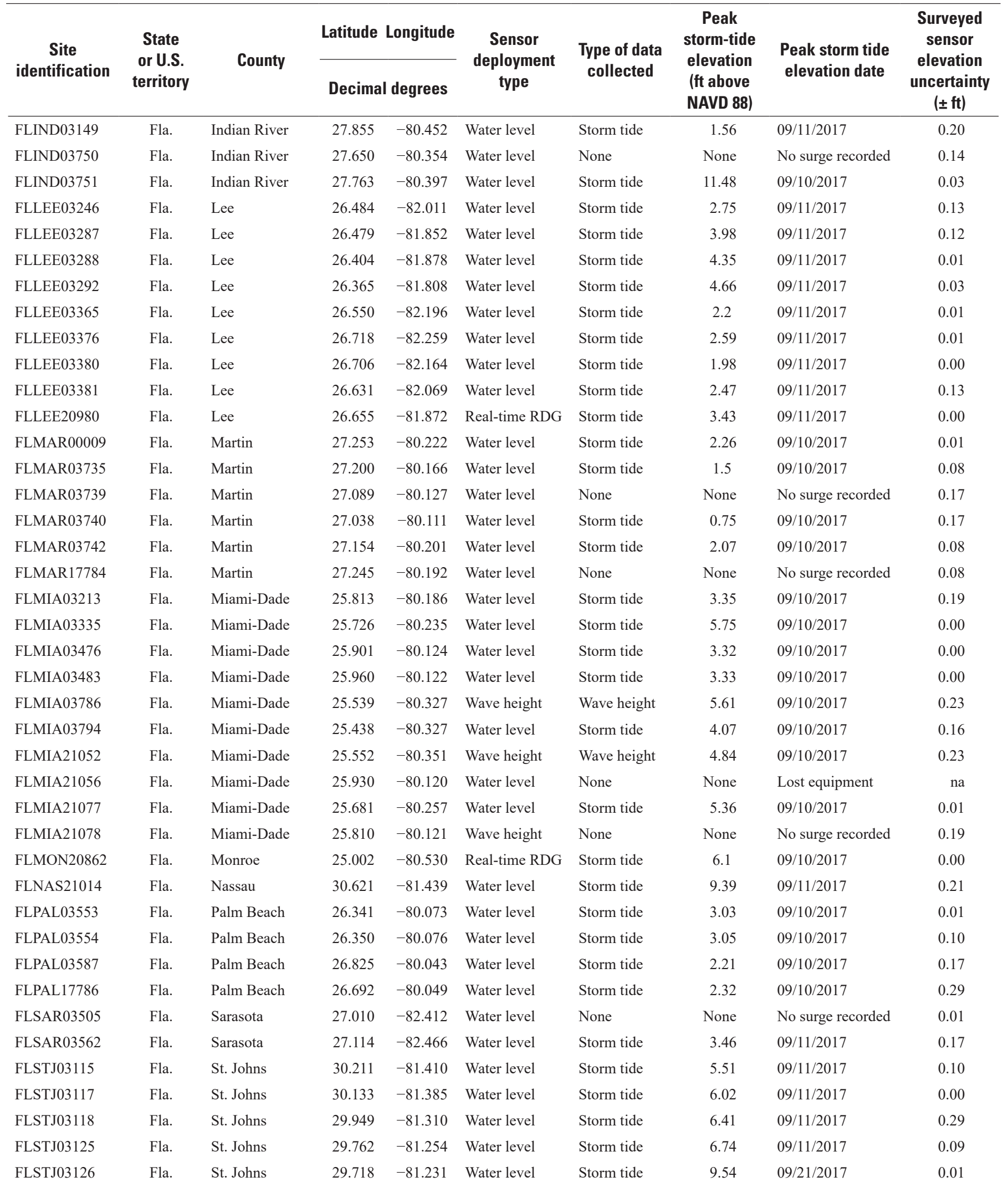


Table 2. Hurricane Irma peak storm-tide and wave-height data for 249 temporarily deployed sites, by State and U.S. territory.-Continued

[Dates shown as month, day, year, referenced to Coordinated Universal Time (UTC). ft, foot; NAVD 88, North American Vertical Datum of 1988; RDG, rapid deployment gage; State or U.S. territory: Fla., Florida; Ga., Georgia; N.C., North Carolina; P.R. Puerto Rico; S.C., South Carolina. \pm , plus or minus; na, not available]

\begin{tabular}{|c|c|c|c|c|c|c|c|c|c|}
\hline \multirow{3}{*}{\begin{tabular}{l}
\multicolumn{1}{c}{$\begin{array}{c}\text { Site } \\
\text { identification }\end{array}$} \\
FLSTJ03129
\end{tabular}} & \multirow{3}{*}{$\begin{array}{c}\begin{array}{c}\text { State } \\
\text { or U.S. } \\
\text { territory }\end{array} \\
\text { Fla. }\end{array}$} & \multirow{3}{*}{\begin{tabular}{|l} 
County \\
St. Johns
\end{tabular}} & \multirow{2}{*}{\multicolumn{2}{|c|}{$\begin{array}{c}\text { Latitude Longitude } \\
\text { Decimal degrees }\end{array}$}} & \multirow{3}{*}{$\begin{array}{c}\begin{array}{c}\text { Sensor } \\
\text { deployment } \\
\text { type }\end{array} \\
\text { Water level }\end{array}$} & \multirow{3}{*}{$\begin{array}{c}\text { Type of data } \\
\text { collected }\end{array}$} & \multirow{3}{*}{$\begin{array}{c}\text { Peak } \\
\text { storm-tide } \\
\text { elevation } \\
\text { (ft above } \\
\text { NAVD 88) } \\
6.27\end{array}$} & \multirow{3}{*}{$\begin{array}{l}\text { Peak storm tide } \\
\text { elevation date }\end{array}$} & \multirow{3}{*}{$\begin{array}{c}\begin{array}{c}\text { Surveyed } \\
\text { sensor } \\
\text { elevation } \\
\text { uncertainty } \\
( \pm \mathrm{ft})\end{array} \\
0.22\end{array}$} \\
\hline & & & & & & & & & \\
\hline & & & 29.680 & -81.221 & & & & & \\
\hline FLSTJ17848 & Fla. & St. Johns & 29.886 & -81.286 & Water level & Storm tide & 6.75 & $09 / 11 / 2017$ & 0.29 \\
\hline FLSTL03729 & Fla. & St. Lucie & 27.363 & -80.249 & Water level & Storm tide & 2.08 & 09/10/2017 & 0.00 \\
\hline FLSTL03731 & Fla. & St. Lucie & 27.527 & -80.348 & Water level & Storm tide & 2.61 & 09/11/2017 & 0.16 \\
\hline FLSTL03732 & Fla. & St. Lucie & 27.467 & -80.330 & Water level & Storm tide & 2.68 & $09 / 11 / 2017$ & 0.00 \\
\hline FLSTL17773 & Fla. & St. Lucie & 27.293 & -80.251 & Water level & Storm tide & 2.06 & 09/10/2017 & 0.01 \\
\hline FLVOL03141 & Fla. & Volusia & 29.147 & -80.965 & Water level & None & None & No surge recorded & 0.01 \\
\hline FLVOL03145 & Fla. & Volusia & 29.031 & -80.916 & Water level & Storm tide & 1.28 & 09/11/2017 & 0.01 \\
\hline FLVOL03146 & Fla. & Volusia & 29.009 & -80.878 & Water level & Storm tide & 8.49 & $09 / 11 / 2017$ & 0.15 \\
\hline FLVOL21027 & Fla. & Volusia & 29.079 & -80.919 & Wave height & Wave height & 9.75 & $09 / 11 / 2017$ & 0.08 \\
\hline FLVOL21104 & Fla. & Volusia & 29.079 & -80.919 & Wave height & Wave height & 9.1 & 09/11/2017 & 0.08 \\
\hline FLVOL21105 & Fla. & Volusia & 29.079 & -80.920 & Wave height & None & None & No surge recorded & 0.08 \\
\hline FLVOL21107 & Fla. & Volusia & 29.079 & -80.920 & Wave height & None & None & No surge recorded & 0.08 \\
\hline FLVOL21109 & Fla. & Volusia & 29.079 & -80.919 & Wave height & Wave height & 9.71 & $09 / 11 / 2017$ & 0.08 \\
\hline GABRY17883 & $\mathrm{Ga}$. & Bryan & 31.791 & -81.202 & Water level & Storm tide & 8.93 & $09 / 11 / 2017$ & 0.02 \\
\hline GACAM17833 & Ga. & Camden & 30.849 & -81.635 & Water level & Storm tide & 7.45 & 09/11/2017 & 0.02 \\
\hline GACAM 17840 & Ga. & Camden & 30.845 & -81.560 & Water level & Storm tide & 6.86 & 09/11/2017 & 0.02 \\
\hline GACAM17842 & $\mathrm{Ga}$. & Camden & 30.771 & -81.581 & Water level & Storm tide & 8.18 & 09/11/2017 & 0.06 \\
\hline GACAM17853 & $\mathrm{Ga}$. & Camden & 31.035 & -81.640 & Water level & Storm tide & 7.29 & $09 / 11 / 2017$ & 0.02 \\
\hline GACAM21130 & Ga. & Camden & 30.745 & -81.600 & Water level & Storm tide & 7.11 & $09 / 11 / 2017$ & 0.02 \\
\hline GACAM21305 & Ga. & Camden & 31.077 & -81.727 & Water level & Storm tide & 7.39 & 09/11/2017 & 0.07 \\
\hline GACHA21079 & Ga. & Chatham & 31.993 & -81.056 & Real-time RDG & Storm tide & 8.64 & 09/11/2017 & 0.04 \\
\hline GACHA21092 & $\mathrm{Ga}$. & Chatham & 32.035 & -80.987 & Real-time RDG & Storm tide & 8.41 & 09/11/2017 & 0.02 \\
\hline GACHA21288 & $\mathrm{Ga}$. & Chatham & 32.002 & -81.200 & Real-time RDG & Storm tide & 7.68 & 09/11/2017 & 0.01 \\
\hline GACHA17815 & Ga. & Chatham & 31.992 & -80.847 & Water level & Storm tide & 8.77 & $09 / 11 / 2017$ & 0.15 \\
\hline GACHA17816 & Ga. & Chatham & 32.006 & -80.842 & Water level & Storm tide & 10.83 & $09 / 11 / 2017$ & 0.02 \\
\hline GACHA17817 & Ga. & Chatham & 32.008 & -80.849 & Water level & Storm tide & 7.98 & 09/11/2017 & 0.02 \\
\hline GACHA17818 & $\mathrm{Ga}$. & Chatham & 32.008 & -80.852 & Water level & Storm tide & 8.02 & $09 / 11 / 2017$ & 0.15 \\
\hline GACHA17820 & Ga. & Chatham & 32.018 & -80.851 & Water level & Storm tide & 7.99 & $09 / 11 / 2017$ & 0.02 \\
\hline GACHA17824 & Ga. & Chatham & 32.021 & -80.899 & Water level & Storm tide & 8.53 & $09 / 11 / 2017$ & 0.02 \\
\hline
\end{tabular}


Table 2. Hurricane Irma peak storm-tide and wave-height data for 249 temporarily deployed sites, by State and U.S. territory.-Continued

[Dates shown as month, day, year, referenced to Coordinated Universal Time (UTC). ft, foot; NAVD 88, North American Vertical Datum of 1988; RDG, rapid deployment gage; State or U.S. territory: Fla., Florida; Ga., Georgia; N.C., North Carolina; P.R. Puerto Rico; S.C., South Carolina. \pm , plus or minus; na, not available]

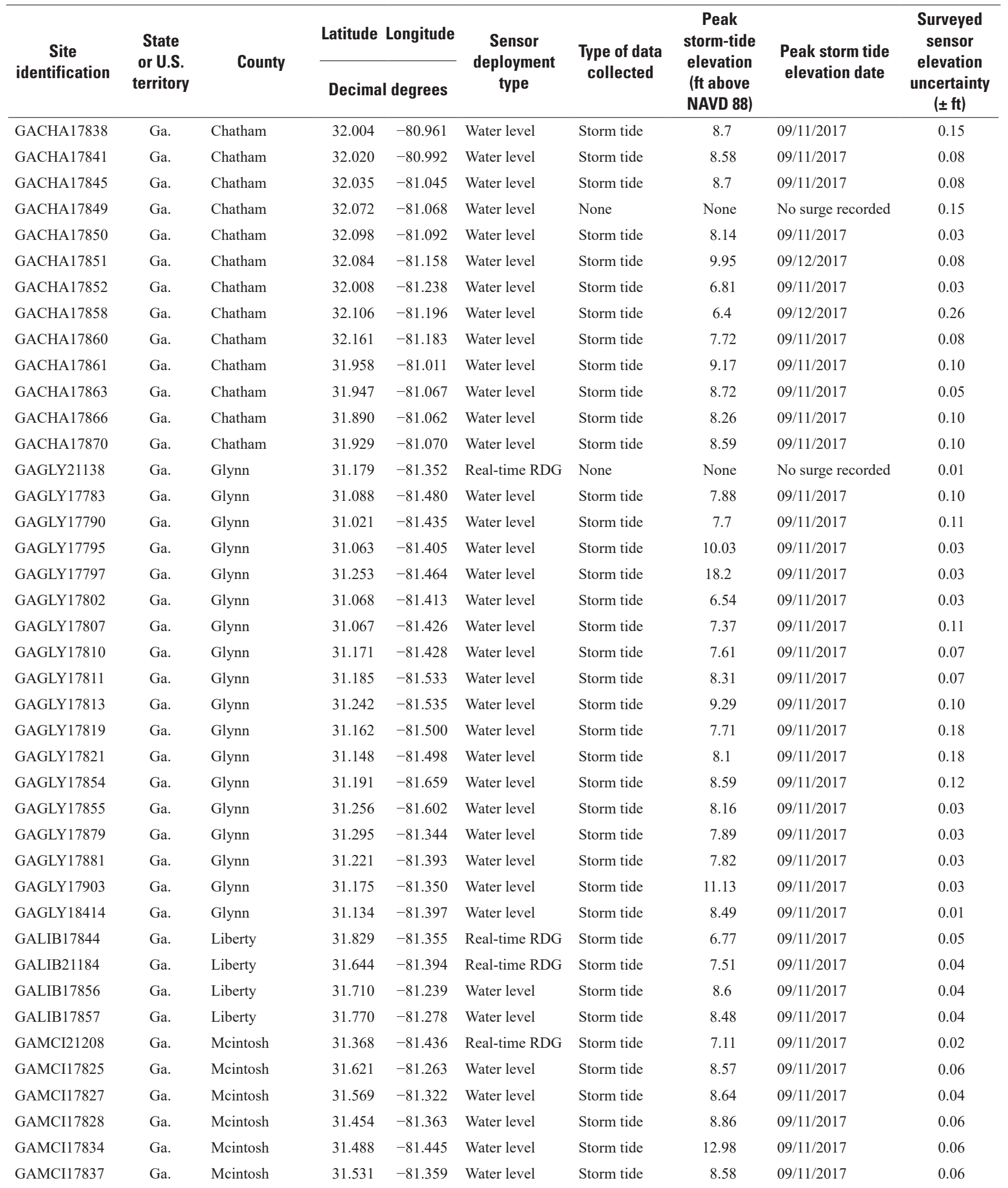


Table 2. Hurricane Irma peak storm-tide and wave-height data for 249 temporarily deployed sites, by State and U.S. territory.-Continued

[Dates shown as month, day, year, referenced to Coordinated Universal Time (UTC). ft, foot; NAVD 88, North American Vertical Datum of 1988; RDG, rapid deployment gage; State or U.S. territory: Fla., Florida; Ga., Georgia; N.C., North Carolina; P.R. Puerto Rico; S.C., South Carolina. \pm , plus or minus; na, not available]

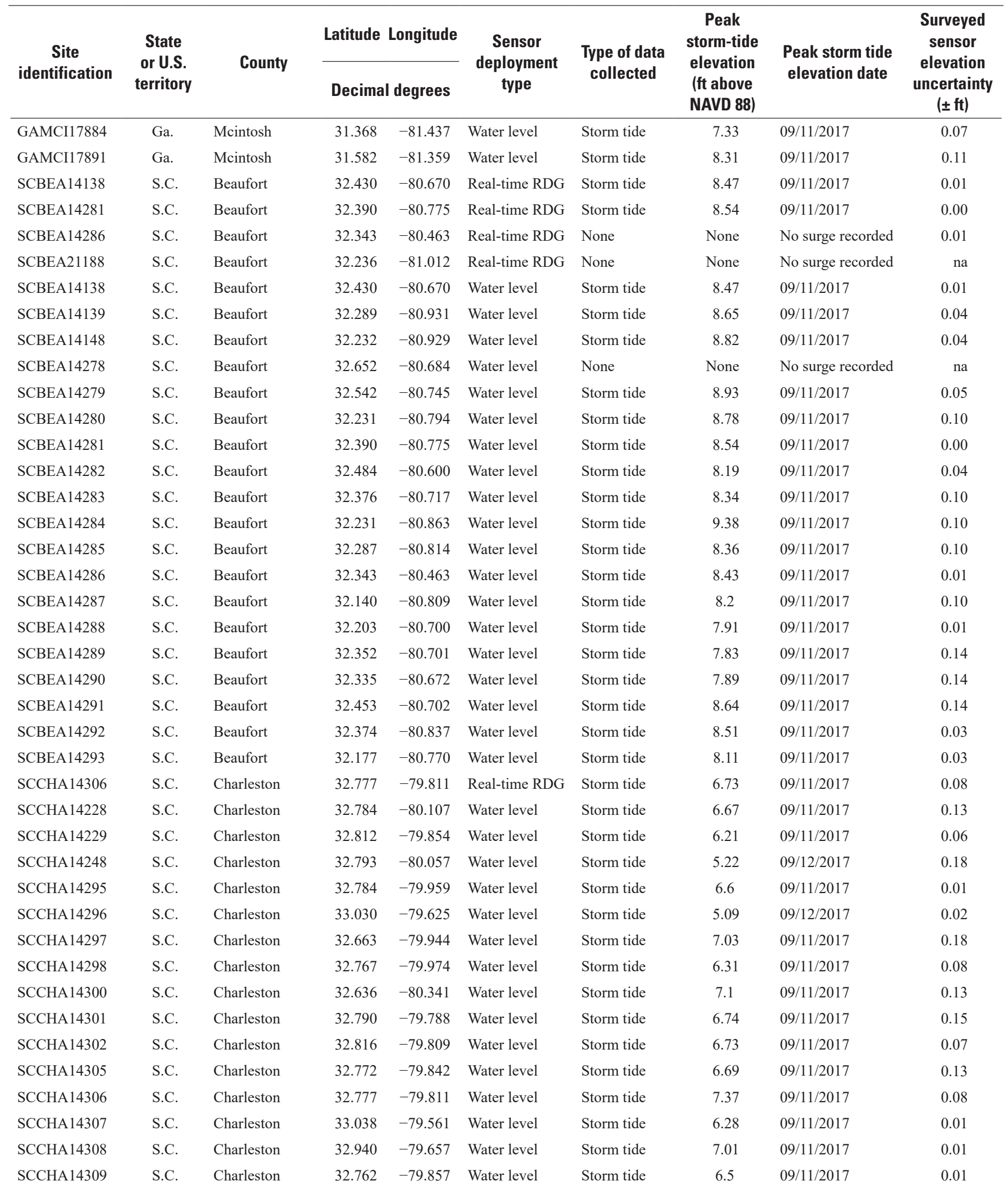


Table 2. Hurricane Irma peak storm-tide and wave-height data for 249 temporarily deployed sites, by State and U.S. territory.-Continued

[Dates shown as month, day, year, referenced to Coordinated Universal Time (UTC). ft, foot; NAVD 88, North American Vertical Datum of 1988; RDG, rapid deployment gage; State or U.S. territory: Fla., Florida; Ga., Georgia; N.C., North Carolina; P.R. Puerto Rico; S.C., South Carolina. \pm , plus or minus; na, not available]

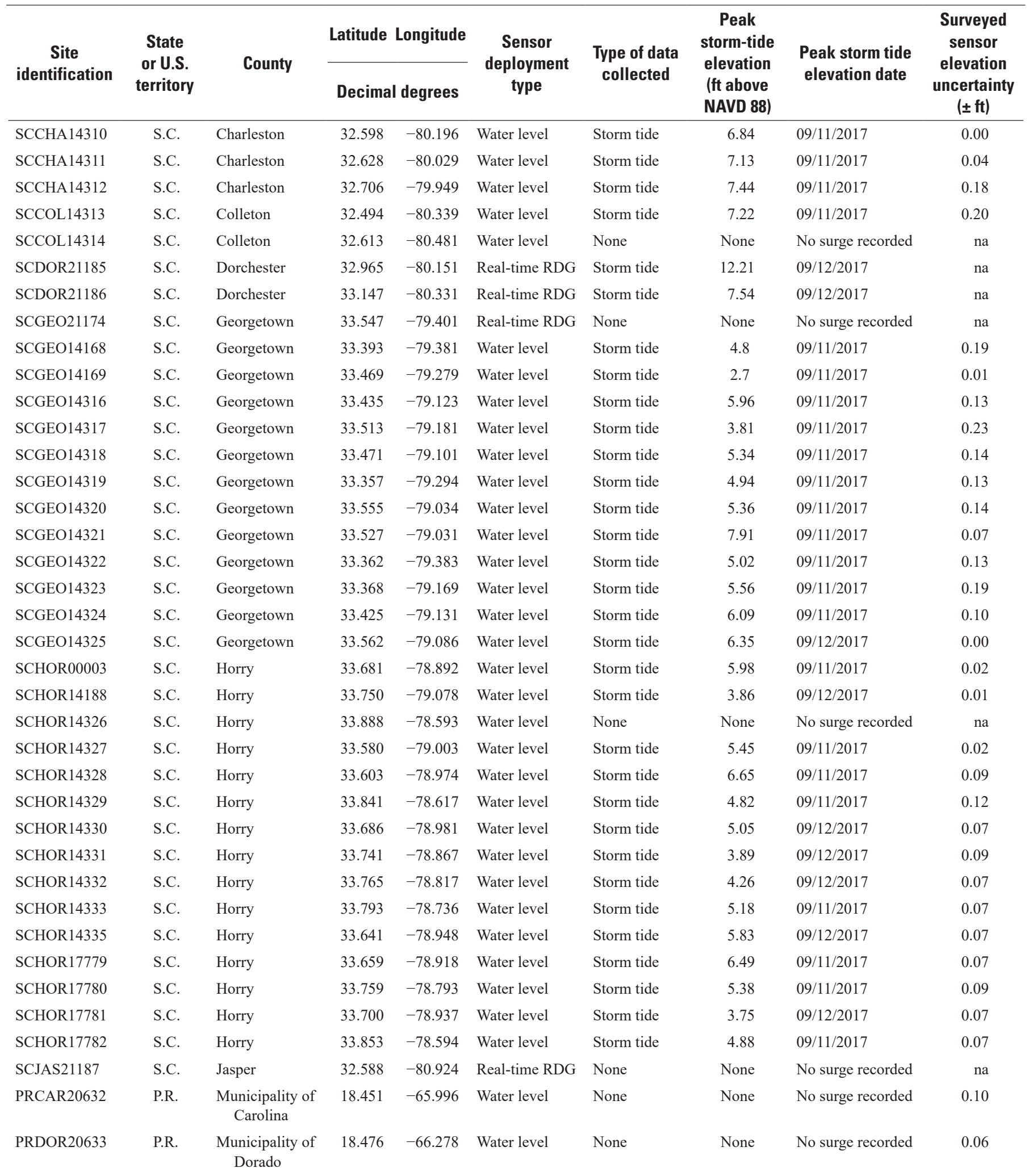


Table 2. Hurricane Irma peak storm-tide and wave-height data for 249 temporarily deployed sites, by State and U.S. territory.-Continued

[Dates shown as month, day, year, referenced to Coordinated Universal Time (UTC). ft, foot; NAVD 88, North American Vertical Datum of 1988; RDG, rapid deployment gage; State or U.S. territory: Fla., Florida; Ga., Georgia; N.C., North Carolina; P.R. Puerto Rico; S.C., South Carolina. \pm , plus or minus; na, not available]

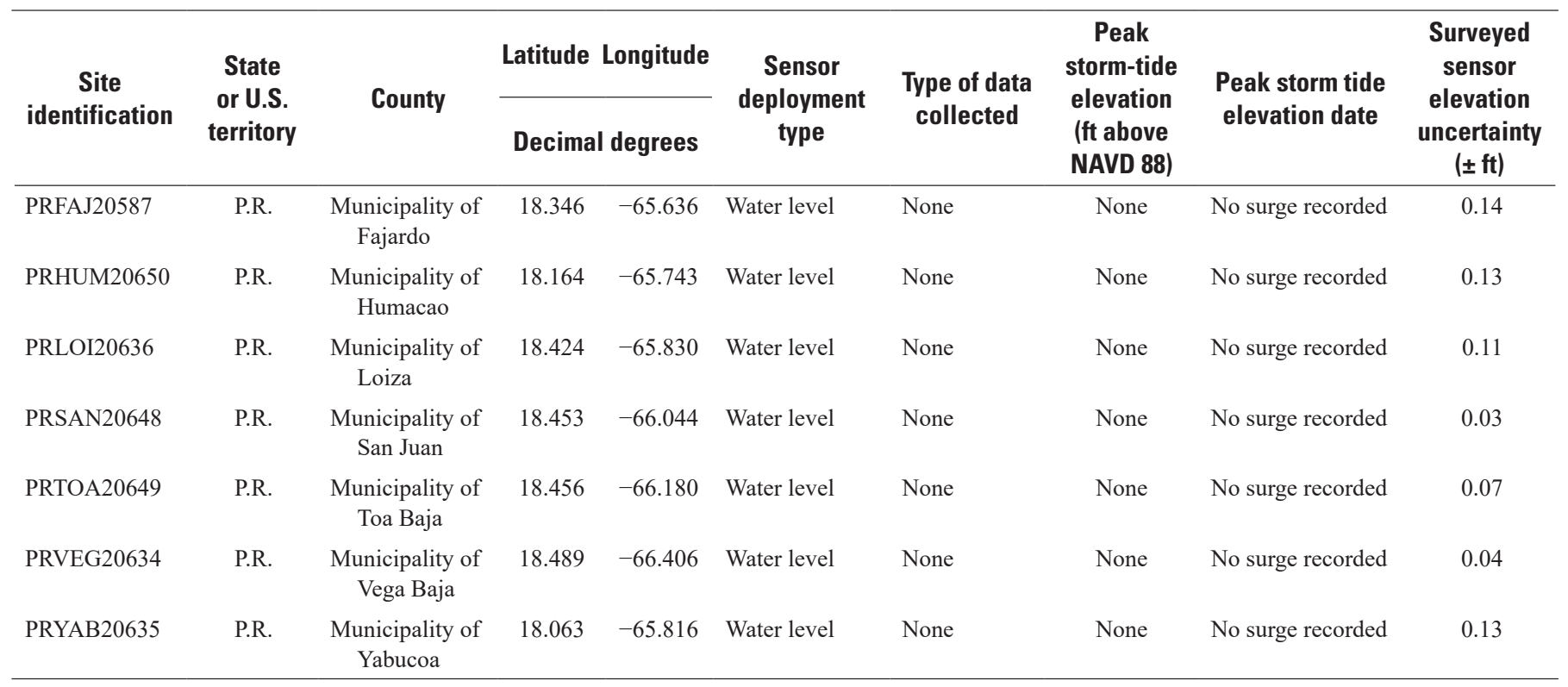


Table 3. Hurricane Irma peak storm-tide data recorded at U.S. Geological Survey long-term monitoring sites, by State and U.S. territory.

[Dates shown as month, day, year, referenced to Coordinated Universal Time (UTC). Datum: NAVD 88, North American Vertical Datum of 1988; NGVD 29, National Geodetic Vertical Datum of 1929; PRVD02, Puerto Rico Vertical Datum of 2002]

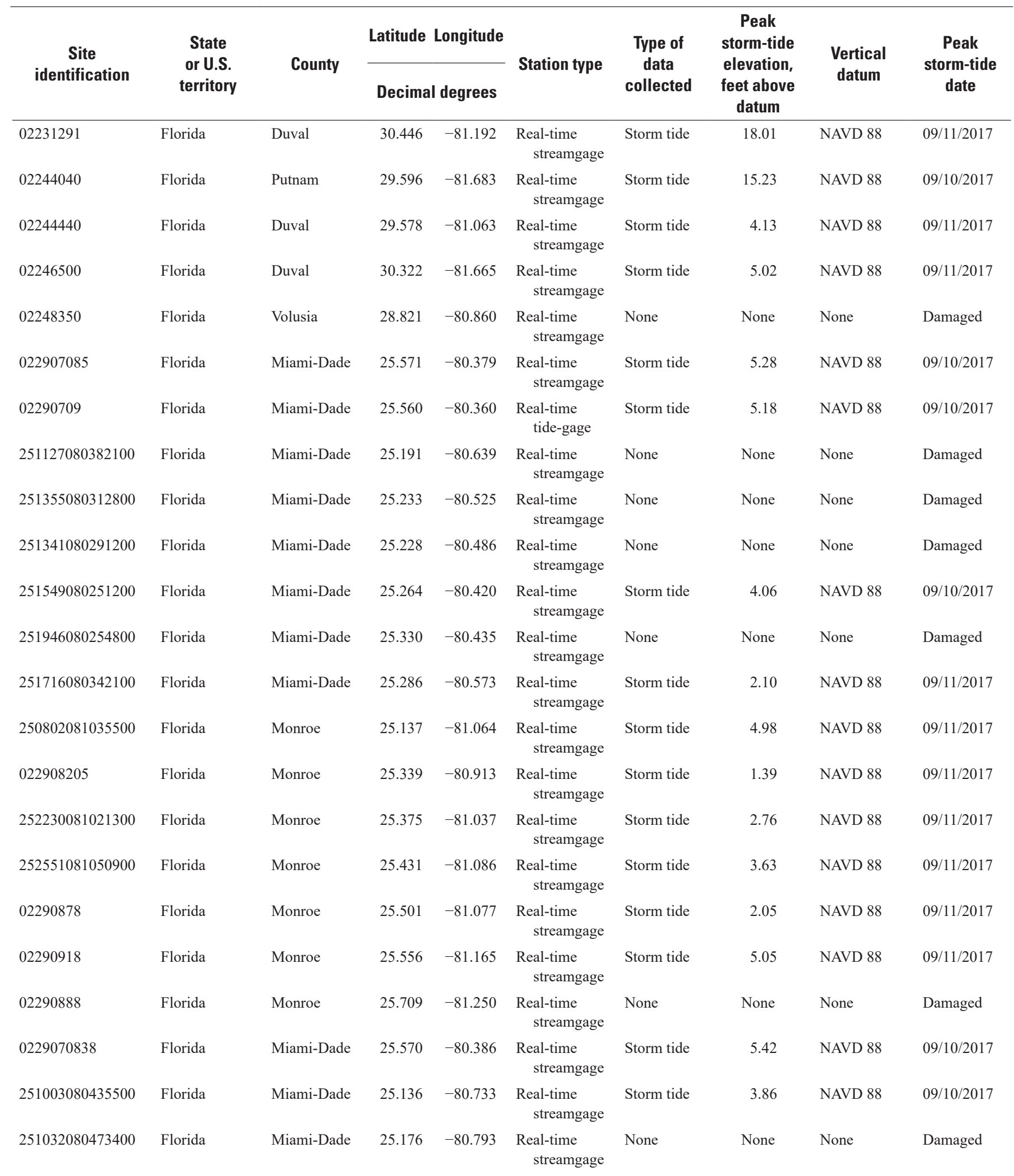


Table 3. Hurricane Irma peak storm-tide data recorded at U.S. Geological Survey long-term monitoring sites, by State and U.S. territory.-Continued

[Dates shown as month, day, year, referenced to Coordinated Universal Time (UTC). Datum: NAVD 88, North American Vertical Datum of 1988; NGVD 29, National Geodetic Vertical Datum of 1929; PRVD02, Puerto Rico Vertical Datum of 2002]

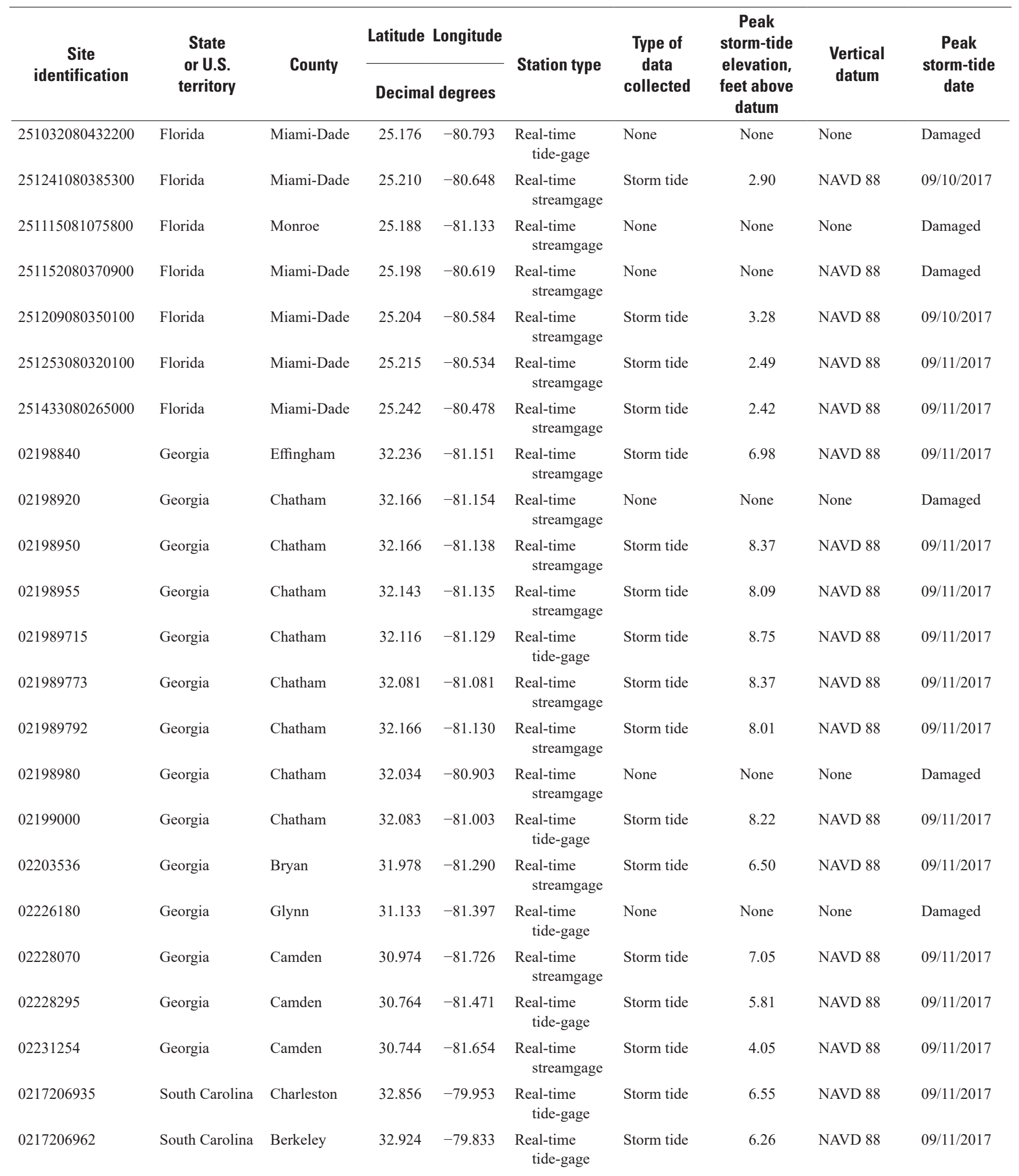


Table 3. Hurricane Irma peak storm-tide data recorded at U.S. Geological Survey long-term monitoring sites, by State and U.S. territory.-Continued

[Dates shown as month, day, year, referenced to Coordinated Universal Time (UTC). Datum: NAVD 88, North American Vertical Datum of 1988; NGVD 29, National Geodetic Vertical Datum of 1929; PRVD02, Puerto Rico Vertical Datum of 2002]

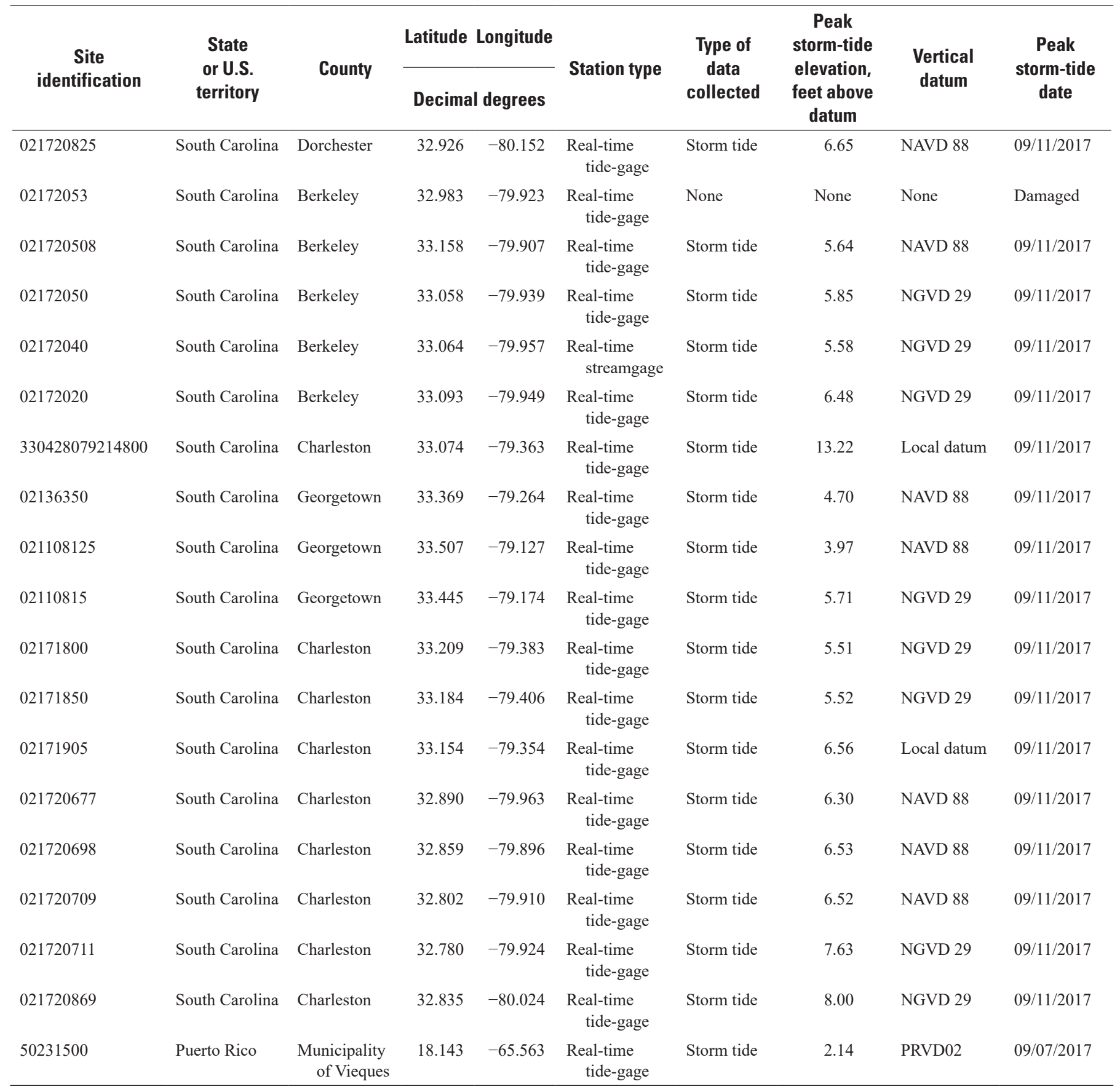


Table 4. Hurricane Irma peak storm-tide data recorded at State and other Federal agency monitoring sites, by State and U.S. territory.

[Dates are shown as month, day, year, referenced to Coordinated Universal Time (UTC). ft, foot; Datums: NAVD 88, North American Vertical Datum of 1988; MSL, Mean Sea Level. NOAA, National Oceanic and Atmospheric Administration; NCDEM, North Carolina Division of Emergency Management. States and U.S. territories: Fla., Florida; Ga., Georgia; N.C., North Carolina; S.C., South Carolina; P.R. Puerto Rico; V.I. Virgin Islands]

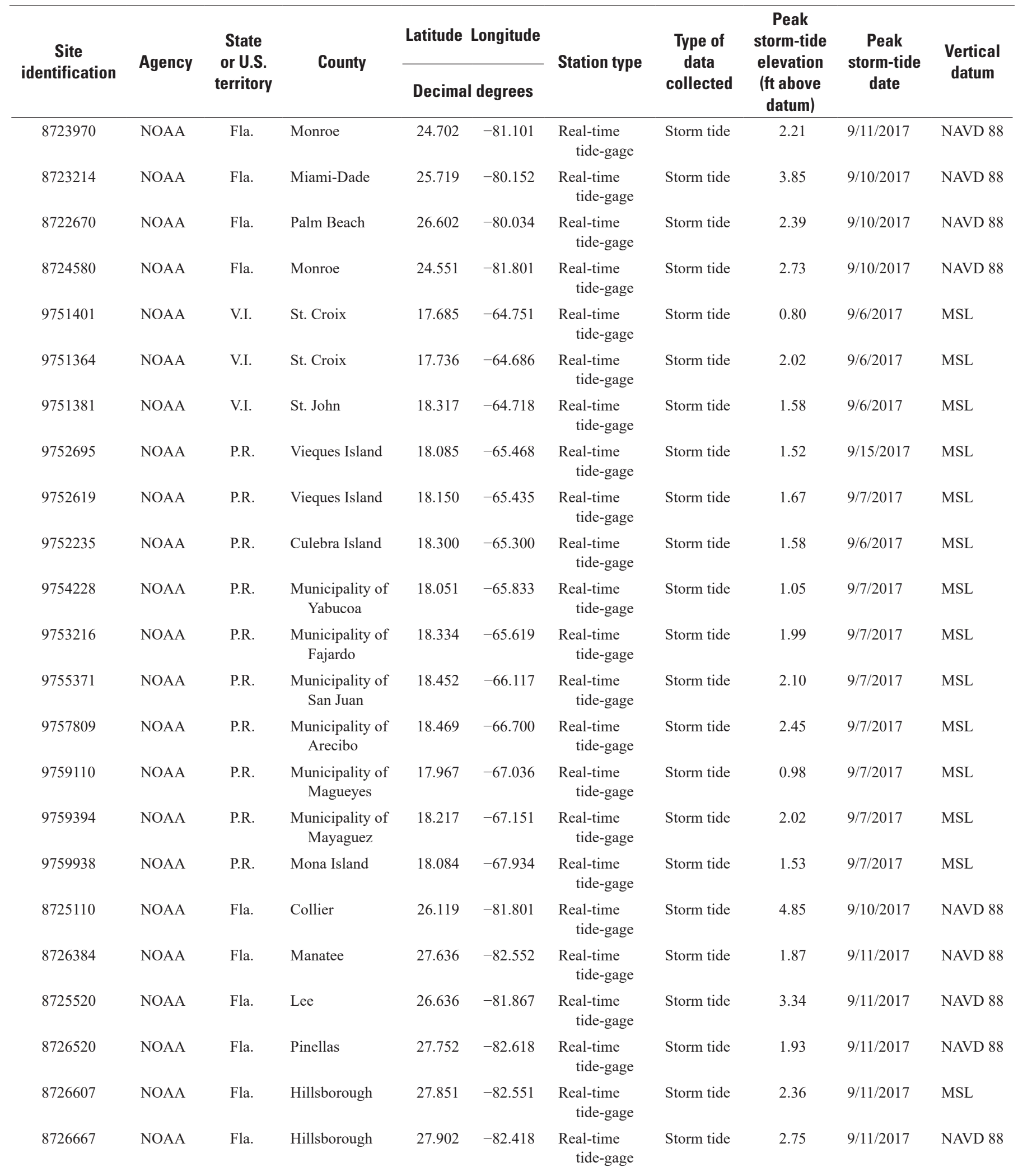


Table 4. Hurricane Irma peak storm-tide data recorded at State and other Federal agency monitoring sites, by State and U.S. territory.-Continued

[Dates are shown as month, day, year, referenced to Coordinated Universal Time (UTC). ft, foot; Datums: NAVD 88, North American Vertical Datum of 1988; MSL, Mean Sea Level. NOAA, National Oceanic and Atmospheric Administration; NCDEM, North Carolina Division of Emergency Management. States and U.S. territories: Fla., Florida; Ga., Georgia; N.C., North Carolina; S.C., South Carolina; P.R. Puerto Rico; V.I. Virgin Islands]

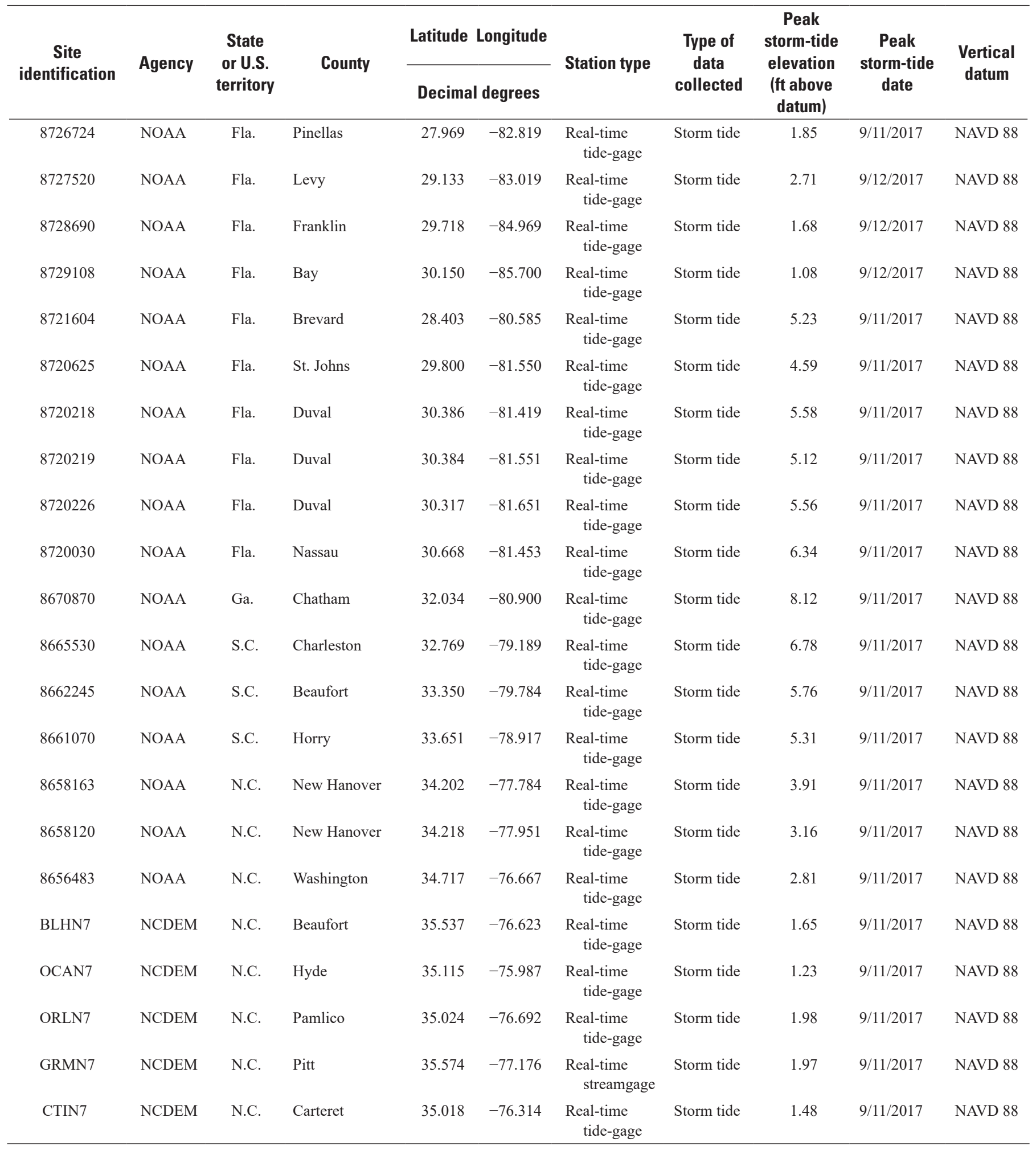


Table 5. Number of sites equipped to monitor Hurricane Irma storm tide, by State and U.S. territory.

[Dates are shown as month, day, year, referenced to Coordinated Universal Time (UTC). HWM, high-water mark; ft, foot. State or U.S. territory: Fla., Florida; Ga., Georgia; N.C., North Carolina; S.C., South Carolina, P.R. Puerto Rico. Datums: NAVD 88, North American Vertical Datum of 1988; NGVD 29, National Geodetic Vertical Datum of 1929; PRVD02, Puerto Rico Vertical Datum of 2002. na, not available]

\begin{tabular}{|c|c|c|c|c|c|c|c|}
\hline \multirow{2}{*}{$\begin{array}{c}\text { Site } \\
\text { identification }\end{array}$} & \multirow{2}{*}{$\begin{array}{c}\text { State } \\
\text { or U.S. } \\
\text { territory }\end{array}$} & \multirow{2}{*}{ County } & Latitude & Longitude & \multirow{2}{*}{$\begin{array}{c}\text { HWM } \\
\text { surveyed } \\
\text { elevation } \\
\text { (ft above datum) }\end{array}$} & \multirow{2}{*}{$\begin{array}{c}\text { Peak } \\
\text { storm-tide } \\
\text { estimated date }\end{array}$} & \multirow{2}{*}{$\begin{array}{c}\text { Vertical datum or } \\
\text { reference point }\end{array}$} \\
\hline & & & \multicolumn{2}{|c|}{ Decimal degrees } & & & \\
\hline FLBRO03524 & Fla. & Broward & 26.261 & -80.084 & 1.5 & 09/11/2017 & NAVD 88 \\
\hline FLBRO20894 & Fla. & Broward & 26.191 & -80.107 & 2.9 & $09 / 11 / 2017$ & NAVD 88 \\
\hline FLCHA23115 & Fla. & Charlotte & 26.999 & -81.888 & 15.7 & 09/11/2017 & NAVD 88 \\
\hline FLCHA23121 & Fla. & Charlotte & 26.976 & -81.896 & 10.3 & 09/11/2017 & NAVD 88 \\
\hline FLCHA23137 & Fla. & Charlotte & 26.975 & -81.886 & 11.3 & $09 / 11 / 2017$ & NAVD 88 \\
\hline FLCLA22832 & Fla. & Clay & 30.040 & -81.708 & 5.3 & $09 / 11 / 2017$ & NAVD 88 \\
\hline FLCLA22849 & Fla. & Clay & 30.082 & -81.809 & 10.3 & 09/11/2017 & NAVD 88 \\
\hline FLCLA22868 & Fla. & Clay & 30.073 & -81.844 & 16.3 & $09 / 11 / 2017$ & NAVD 88 \\
\hline FLCLA22871 & Fla. & Clay & 30.073 & -81.851 & 17.0 & 09/11/2017 & NAVD 88 \\
\hline FLCLA22888 & Fla. & Clay & 30.061 & -81.875 & 20.3 & $09 / 11 / 2017$ & NAVD 88 \\
\hline FLCLA22896 & Fla. & Clay & 30.060 & -81.870 & 19.9 & 09/11/2017 & NAVD 88 \\
\hline FLCLA22896 & Fla. & Clay & 30.060 & -81.870 & 19.9 & 09/11/2017 & NAVD 88 \\
\hline FLCLA22958 & Fla. & Clay & 30.075 & -81.867 & 17.5 & $09 / 11 / 2017$ & NAVD 88 \\
\hline FLCLA22962 & Fla. & Clay & 30.080 & -81.878 & 19.7 & 09/11/2017 & NAVD 88 \\
\hline FLCLA22980 & Fla. & Clay & 30.086 & -81.880 & 19.9 & 09/11/2017 & NAVD 88 \\
\hline FLCLA22980 & Fla. & Clay & 30.085 & -81.880 & 19.9 & 09/11/2017 & NAVD 88 \\
\hline FLCLA23098 & Fla. & Clay & 30.066 & -81.859 & 18.0 & $09 / 11 / 2017$ & NAVD 88 \\
\hline FLCLA23103 & Fla. & Clay & 30.076 & -81.858 & 17.8 & $09 / 11 / 2017$ & NAVD 88 \\
\hline FLCLA23107 & Fla. & Clay & 30.090 & -81.894 & 22.2 & $09 / 11 / 2017$ & NAVD 88 \\
\hline FLCLA23114 & Fla. & Clay & 30.077 & -81.873 & 19.4 & 09/11/2017 & NAVD 88 \\
\hline FLCLA23119 & Fla. & Clay & 30.042 & -81.869 & 24.3 & 09/11/2017 & NAVD 88 \\
\hline FLCLA23125 & Fla. & Clay & 30.052 & -81.873 & 23.5 & 09/11/2017 & NAVD 88 \\
\hline FLCLA23129 & Fla. & Clay & 30.009 & -81.858 & 31.8 & $09 / 11 / 2017$ & NAVD 88 \\
\hline FLCLA23146 & Fla. & Clay & 30.069 & -81.818 & 12.3 & 09/11/2017 & NAVD 88 \\
\hline FLCLA23147 & Fla. & Clay & 30.070 & -81.734 & 5.3 & 09/11/2017 & NAVD 88 \\
\hline FLCLA23157 & Fla. & Clay & 30.083 & -81.787 & 4.6 & 09/11/2017 & Above ground level \\
\hline FLCLA23153 & Fla. & Clay & 30.114 & -81.767 & 5.9 & $09 / 11 / 2017$ & NAVD 88 \\
\hline FLCLA23159 & Fla. & Clay & 30.085 & -81.797 & 8.9 & $09 / 11 / 2017$ & NAVD 88 \\
\hline FLCLA23162 & Fla. & Clay & 29.993 & -81.852 & 35.1 & $09 / 11 / 2017$ & NAVD 88 \\
\hline FLCLA23163 & Fla. & Clay & 30.018 & -81.860 & 29.8 & $09 / 11 / 2017$ & NAVD 88 \\
\hline
\end{tabular}


Table 5. Number of sites equipped to monitor Hurricane Irma storm tide, by State and U.S. territory.-Continued

[Dates are shown as month, day, year, referenced to Coordinated Universal Time (UTC). HWM, high-water mark; ft, foot. State or U.S. territory: Fla., Florida; Ga., Georgia; N.C., North Carolina; S.C., South Carolina, P.R. Puerto Rico. Datums: NAVD 88, North American Vertical Datum of 1988; NGVD 29, National Geodetic Vertical Datum of 1929; PRVD02, Puerto Rico Vertical Datum of 2002. na, not available]

\begin{tabular}{|c|c|c|c|c|c|c|c|}
\hline \multirow{2}{*}{$\begin{array}{c}\text { Site } \\
\text { identification }\end{array}$} & \multirow{2}{*}{$\begin{array}{c}\text { State } \\
\text { or U.S. } \\
\text { territory }\end{array}$} & \multirow{2}{*}{ County } & Latitude & Longitude & \multirow{2}{*}{$\begin{array}{c}\text { HWM } \\
\text { surveyed } \\
\text { elevation } \\
\text { (ft above datum) }\end{array}$} & \multirow{2}{*}{$\begin{array}{c}\text { Peak } \\
\text { storm-tide } \\
\text { estimated date }\end{array}$} & \multirow{2}{*}{$\begin{array}{l}\text { Vertical datum or } \\
\text { reference point }\end{array}$} \\
\hline & & & \multicolumn{2}{|c|}{ Decimal degrees } & & & \\
\hline FLCLA23166 & Fla. & Clay & 30.038 & -81.881 & 25.0 & 09/11/2017 & NAVD 88 \\
\hline FLCLA23167 & Fla. & Clay & 30.118 & -81.760 & 6.1 & $09 / 11 / 2017$ & NAVD 88 \\
\hline FLCOL03237 & Fla. & Collier & 25.845 & -81.387 & 8.8 & 09/11/2017 & NAVD 88 \\
\hline FLCOL21927 & Fla. & Collier & 25.850 & -81.385 & 7.8 & 09/11/2017 & NAVD 88 \\
\hline FLCOL21928 & Fla. & Collier & 25.849 & -81.385 & 8.1 & 09/11/2017 & NAVD 88 \\
\hline FLCOL03089 & Fla. & Collier & 25.957 & -81.512 & 5.0 & 09/11/2017 & NAVD 88 \\
\hline FLCOL03176 & Fla. & Collier & 25.922 & -81.648 & 7.3 & 09/11/2017 & NAVD 88 \\
\hline FLCOL22502 & Fla. & Collier & 25.927 & -81.544 & 6.3 & 09/11/2017 & NAVD 88 \\
\hline FLCOL22502 & Fla. & Collier & 25.927 & -81.544 & 6.5 & 09/11/2017 & NAVD 88 \\
\hline FLCOL22503 & Fla. & Collier & 25.895 & -81.456 & 5.4 & $09 / 11 / 2017$ & NAVD 88 \\
\hline FLCOL22503 & Fla. & Collier & 25.895 & -81.456 & 5.4 & $09 / 11 / 2017$ & NAVD 88 \\
\hline FLCOL22504 & Fla. & Collier & 25.909 & -81.511 & 6.8 & 09/11/2017 & NAVD 88 \\
\hline FLCOL22506 & Fla. & Collier & 25.872 & -81.382 & na & 09/11/2017 & NAVD 88 \\
\hline FLCOL22585 & Fla. & Collier & 26.222 & -81.813 & 4.3 & 09/11/2017 & NAVD 88 \\
\hline FLCOL22586 & Fla. & Collier & 26.156 & -81.787 & 4.7 & 09/11/2017 & NAVD 88 \\
\hline FLCOL22587 & Fla. & Collier & 26.141 & -81.789 & 4.4 & 09/11/2017 & NAVD 88 \\
\hline FLCOL22592 & Fla. & Collier & 26.212 & -81.812 & 4.6 & 09/11/2017 & NAVD 88 \\
\hline FLCOL22593 & Fla. & Collier & 26.122 & -81.802 & 4.1 & 09/11/2017 & NAVD 88 \\
\hline FLCOL22716 & Fla. & Collier & 26.100 & -81.753 & 3.4 & 09/11/2017 & NAVD 88 \\
\hline FLCOL22717 & Fla. & Collier & 25.971 & -81.736 & 4.9 & 09/11/2017 & NAVD 88 \\
\hline FLCOL22718 & Fla. & Collier & 25.914 & -81.718 & 6.1 & $09 / 11 / 2017$ & NAVD 88 \\
\hline FLCOL22719 & Fla. & Collier & 25.918 & -81.726 & 5.8 & 09/11/2017 & NAVD 88 \\
\hline FLCOL 22720 & Fla. & Collier & 25.910 & -81.697 & 6.5 & $09 / 11 / 2017$ & NAVD 88 \\
\hline FLCOL22721 & Fla. & Collier & 26.126 & -81.774 & 4.4 & 09/11/2017 & NAVD 88 \\
\hline FLCOL22722 & Fla. & Collier & 26.113 & -81.783 & 4.3 & $09 / 11 / 2017$ & NAVD 88 \\
\hline FLCOL22722 & Fla. & Collier & 26.113 & -81.783 & 4.3 & 09/11/2017 & NAVD 88 \\
\hline FLCOL 22723 & Fla. & Collier & 26.025 & -81.731 & 4.0 & 09/11/2017 & NAVD 88 \\
\hline FLCOL22724 & Fla. & Collier & 25.984 & -81.722 & 4.9 & 09/11/2017 & NAVD 88 \\
\hline FLCOL22725 & Fla. & Collier & 25.937 & -81.700 & 5.6 & 09/11/2017 & NAVD 88 \\
\hline FLCOL22726 & Fla. & Collier & 25.933 & -81.657 & 6.1 & 09/11/2017 & NAVD 88 \\
\hline
\end{tabular}


Table 5. Number of sites equipped to monitor Hurricane Irma storm tide, by State and U.S. territory.—Continued

[Dates are shown as month, day, year, referenced to Coordinated Universal Time (UTC). HWM, high-water mark; ft, foot. State or U.S. territory: Fla., Florida; Ga., Georgia; N.C., North Carolina; S.C., South Carolina, P.R. Puerto Rico. Datums: NAVD 88, North American Vertical Datum of 1988; NGVD 29, National Geodetic Vertical Datum of 1929; PRVD02, Puerto Rico Vertical Datum of 2002. na, not available]

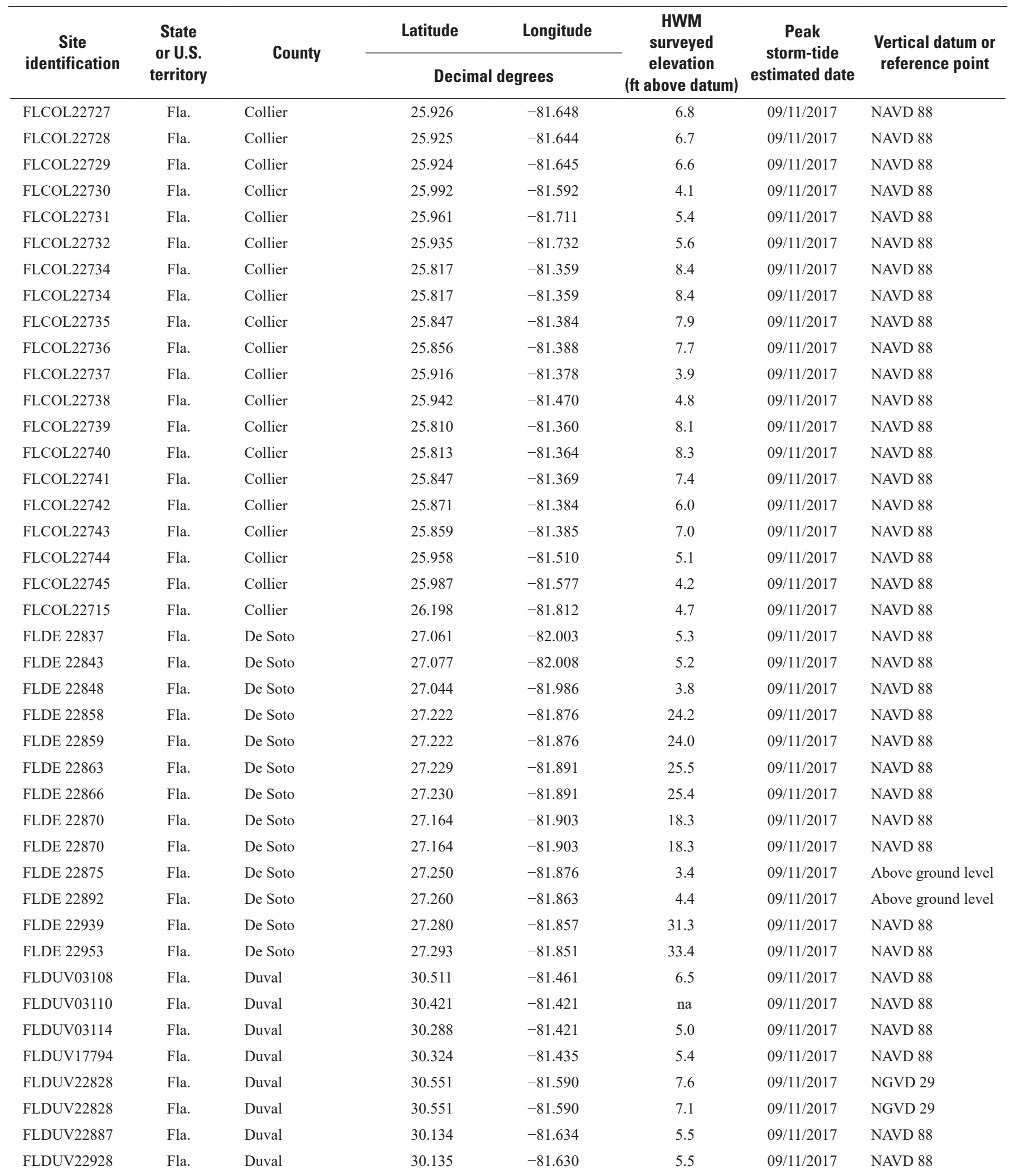


Table 5. Number of sites equipped to monitor Hurricane Irma storm tide, by State and U.S. territory.-Continued

[Dates are shown as month, day, year, referenced to Coordinated Universal Time (UTC). HWM, high-water mark; ft, foot. State or U.S. territory: Fla., Florida; Ga., Georgia; N.C., North Carolina; S.C., South Carolina, P.R. Puerto Rico. Datums: NAVD 88, North American Vertical Datum of 1988; NGVD 29, National Geodetic Vertical Datum of 1929; PRVD02, Puerto Rico Vertical Datum of 2002. na, not available]

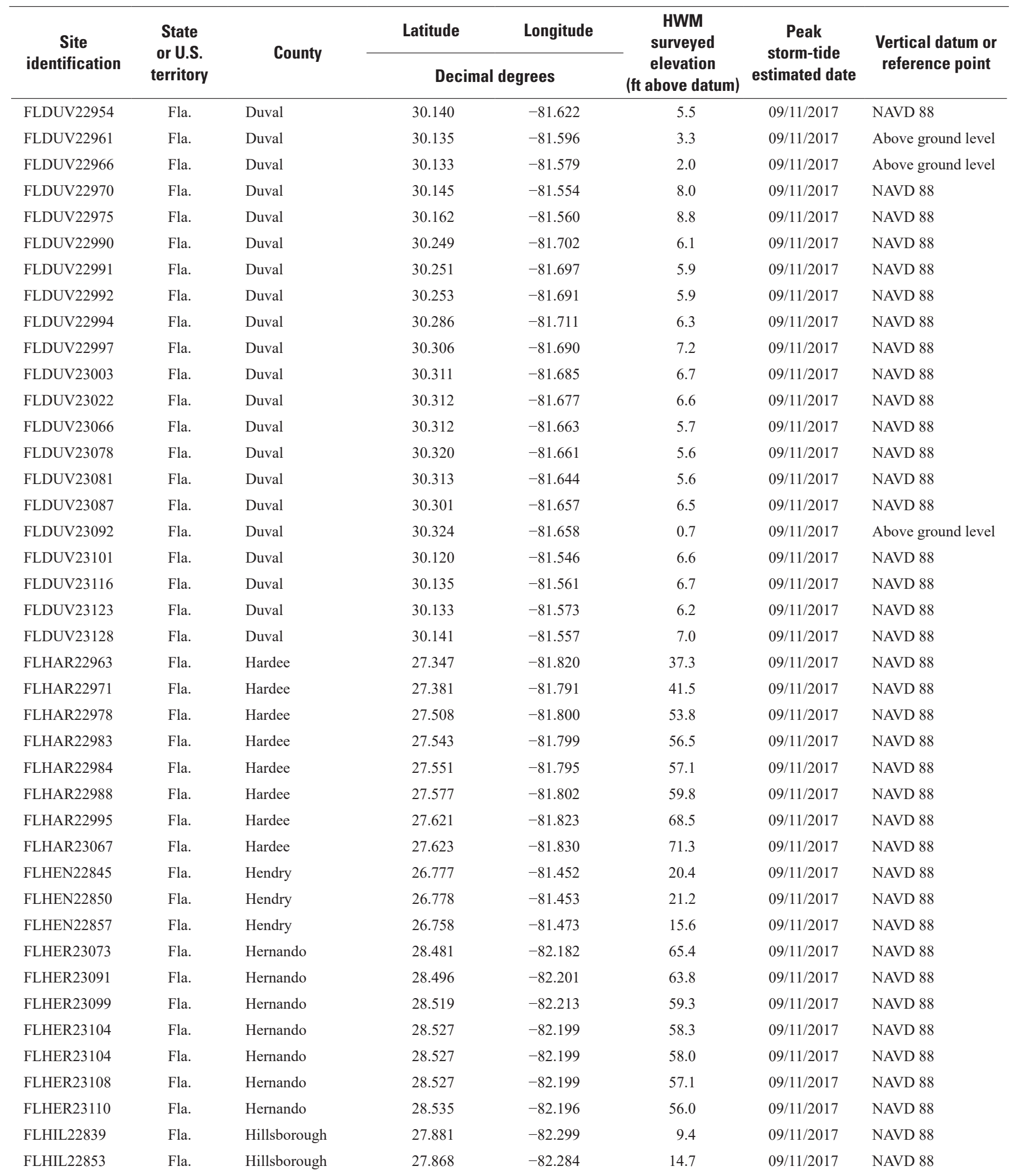


Table 5. Number of sites equipped to monitor Hurricane Irma storm tide, by State and U.S. territory.—Continued

[Dates are shown as month, day, year, referenced to Coordinated Universal Time (UTC). HWM, high-water mark; ft, foot. State or U.S. territory: Fla., Florida; Ga., Georgia; N.C., North Carolina; S.C., South Carolina, P.R. Puerto Rico. Datums: NAVD 88, North American Vertical Datum of 1988; NGVD 29, National Geodetic Vertical Datum of 1929; PRVD02, Puerto Rico Vertical Datum of 2002. na, not available]

\begin{tabular}{|c|c|c|c|c|c|c|c|}
\hline \multirow{2}{*}{$\begin{array}{c}\text { Site } \\
\text { identification }\end{array}$} & \multirow{2}{*}{$\begin{array}{c}\text { State } \\
\text { or U.S. } \\
\text { territory }\end{array}$} & \multirow{2}{*}{ County } & Latitude & Longitude & \multirow{2}{*}{$\begin{array}{c}\text { HWM } \\
\text { surveyed } \\
\text { elevation } \\
\text { (ft above datum) }\end{array}$} & \multirow{2}{*}{$\begin{array}{c}\text { Peak } \\
\text { storm-tide } \\
\text { estimated date }\end{array}$} & \multirow{2}{*}{$\begin{array}{l}\text { Vertical datum or } \\
\text { reference point }\end{array}$} \\
\hline & & & \multicolumn{2}{|c|}{ Decimal degrees } & & & \\
\hline FLHIL22846 & Fla. & Hillsborough & 27.871 & -82.286 & 13.9 & 09/11/2017 & NAVD 88 \\
\hline FLHIL22861 & Fla. & Hillsborough & 27.862 & -82.265 & 22.3 & 09/11/2017 & NAVD 88 \\
\hline FLHIL22867 & Fla. & Hillsborough & 27.869 & -82.286 & 13.7 & $09 / 11 / 2017$ & NAVD 88 \\
\hline FLHIL 22872 & Fla. & Hillsborough & 27.878 & -82.298 & 9.8 & 09/11/2017 & NAVD 88 \\
\hline FLHIL22876 & Fla. & Hillsborough & 27.889 & -82.302 & 7.7 & $09 / 11 / 2017$ & NAVD 88 \\
\hline FLHIL22886 & Fla. & Hillsborough & 27.867 & -82.230 & 24.5 & $09 / 11 / 2017$ & NAVD 88 \\
\hline FLHIL22894 & Fla. & Hillsborough & 27.873 & -82.230 & 27.4 & 09/11/2017 & NAVD 88 \\
\hline FLHIL22846 & Fla. & Hillsborough & 27.871 & -82.286 & 13.9 & 09/11/2017 & NAVD 88 \\
\hline FLIND03751 & Fla. & Indian River & 27.763 & -80.397 & 11.3 & 09/11/2017 & NAVD 88 \\
\hline FLIND03751 & Fla. & Indian River & 27.763 & -80.397 & na & na & NAVD 88 \\
\hline FLIND03149 & Fla. & Indian River & 27.855 & -80.452 & 1.6 & 09/11/2017 & NAVD 88 \\
\hline FLIND03149 & Fla. & Indian River & 27.855 & -80.452 & 1.7 & $09 / 11 / 2017$ & NAVD 88 \\
\hline FLLEE03288 & Fla. & Lee & 26.404 & -81.878 & 4.0 & $09 / 11 / 2017$ & NAVD 88 \\
\hline FLLEE21933 & Fla. & Lee & 26.766 & -82.265 & 3.4 & 09/11/2017 & NAVD 88 \\
\hline FLLEE21935 & Fla. & Lee & 26.766 & -82.265 & 3.1 & 09/11/2017 & NAVD 88 \\
\hline FLLEE03380 & Fla. & Lee & 26.706 & -82.164 & 2.0 & 09/11/2017 & NAVD 88 \\
\hline FLLEE22869 & Fla. & Lee & 26.340 & -81.807 & 4.0 & 09/11/2017 & NAVD 88 \\
\hline FLLEE22874 & Fla. & Lee & 26.341 & -81.799 & 4.2 & 09/11/2017 & NAVD 88 \\
\hline FLLEE22884 & Fla. & Lee & 26.669 & -81.733 & 9.4 & 09/11/2017 & NAVD 88 \\
\hline FLLEE22889 & Fla. & Lee & 26.669 & -81.738 & 9.2 & 09/11/2017 & NAVD 88 \\
\hline FLLEE22891 & Fla. & Lee & 26.341 & -81.790 & 5.0 & $09 / 11 / 2017$ & NAVD 88 \\
\hline FLLEE22895 & Fla. & Lee & 26.673 & -81.744 & 12.8 & 09/11/2017 & NAVD 88 \\
\hline FLLEE22907 & Fla. & Lee & 26.341 & -81.783 & 5.5 & 09/11/2017 & NAVD 88 \\
\hline FLLEE22940 & Fla. & Lee & 26.689 & -81.752 & 5.4 & 09/11/2017 & NAVD 88 \\
\hline FLLEE22951 & Fla. & Lee & 26.343 & -81.778 & 6.3 & 09/11/2017 & NAVD 88 \\
\hline FLLEE22952 & Fla. & Lee & 26.682 & -81.739 & 7.8 & $09 / 11 / 2017$ & NAVD 88 \\
\hline FLLEE22955 & Fla. & Lee & 26.341 & -81.772 & 7.3 & $09 / 11 / 2017$ & NAVD 88 \\
\hline FLLEE22957 & Fla. & Lee & 26.679 & -81.741 & 8.6 & 09/11/2017 & NAVD 88 \\
\hline FLLEE22960 & Fla. & Lee & 26.338 & -81.765 & 10.4 & 09/11/2017 & NAVD 88 \\
\hline FLLEE22965 & Fla. & Lee & 26.335 & -81.762 & 11.6 & 09/11/2017 & NAVD 88 \\
\hline
\end{tabular}


Table 5. Number of sites equipped to monitor Hurricane Irma storm tide, by State and U.S. territory. - Continued

[Dates are shown as month, day, year, referenced to Coordinated Universal Time (UTC). HWM, high-water mark; ft, foot. State or U.S. territory: Fla., Florida; Ga., Georgia; N.C., North Carolina; S.C., South Carolina, P.R. Puerto Rico. Datums: NAVD 88, North American Vertical Datum of 1988; NGVD 29, National Geodetic Vertical Datum of 1929; PRVD02, Puerto Rico Vertical Datum of 2002. na, not available]

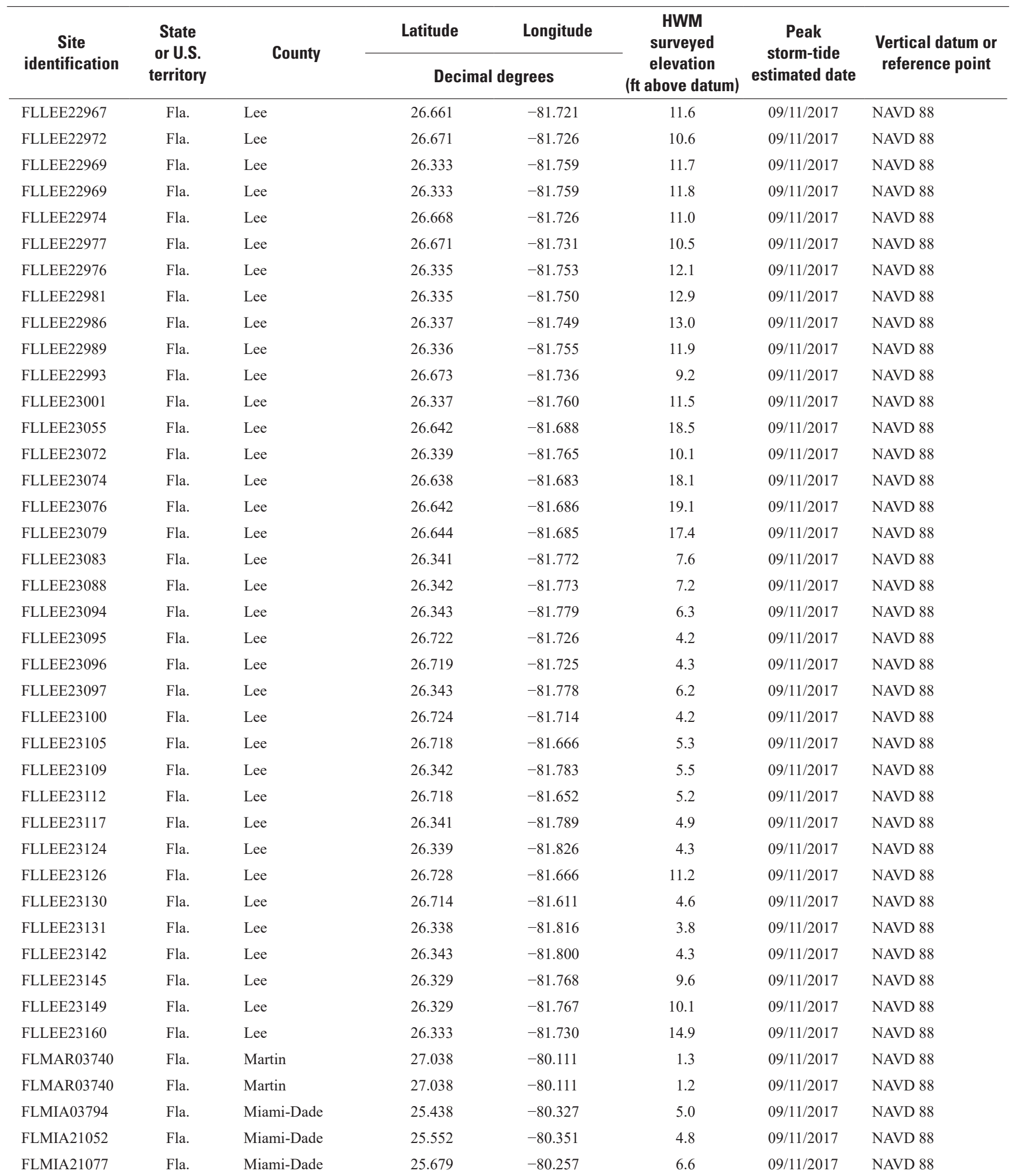


Table 5. Number of sites equipped to monitor Hurricane Irma storm tide, by State and U.S. territory.—Continued

[Dates are shown as month, day, year, referenced to Coordinated Universal Time (UTC). HWM, high-water mark; ft, foot. State or U.S. territory: Fla., Florida; Ga., Georgia; N.C., North Carolina; S.C., South Carolina, P.R. Puerto Rico. Datums: NAVD 88, North American Vertical Datum of 1988; NGVD 29, National Geodetic Vertical Datum of 1929; PRVD02, Puerto Rico Vertical Datum of 2002. na, not available]

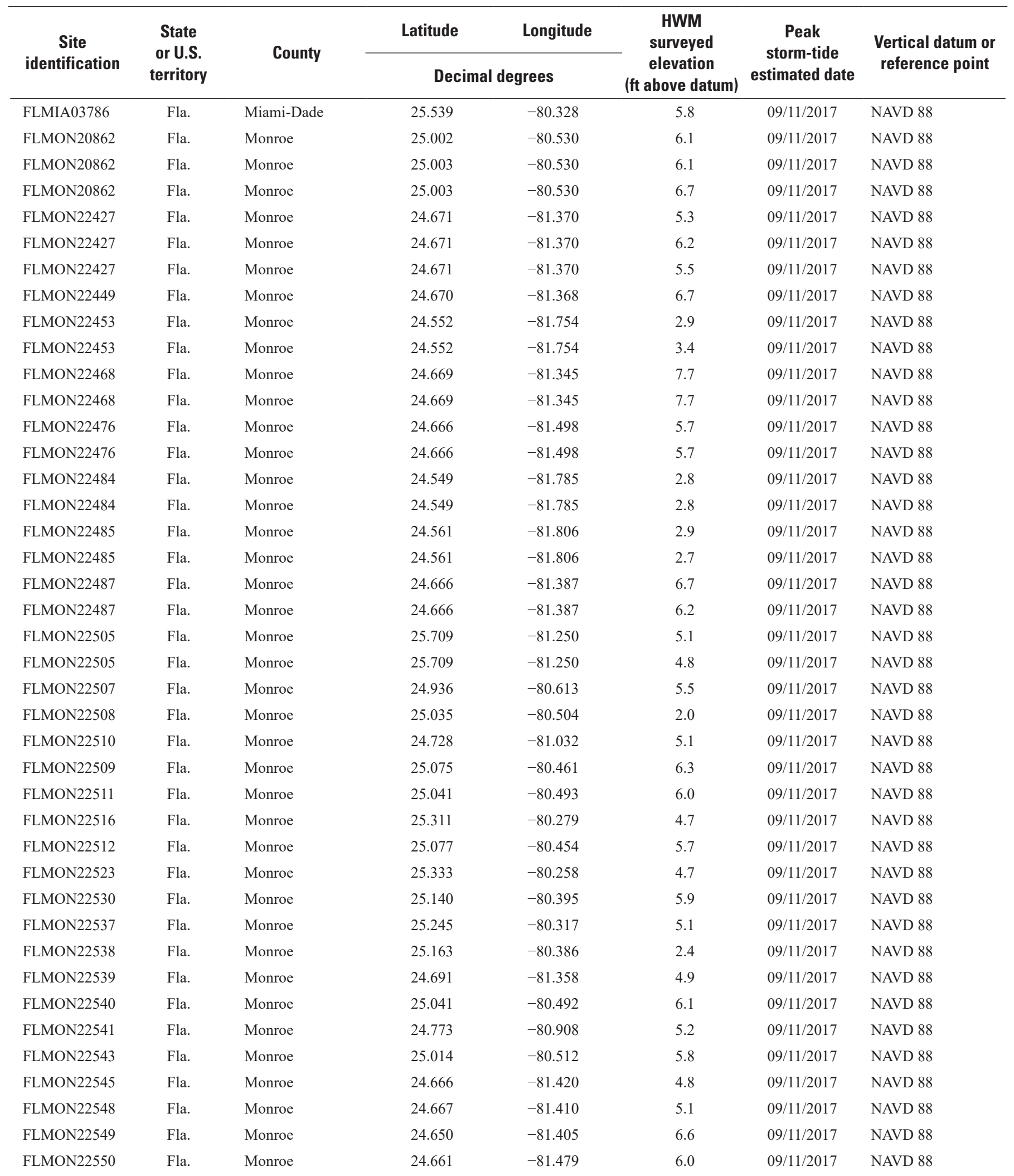


Table 5. Number of sites equipped to monitor Hurricane Irma storm tide, by State and U.S. territory. - Continued

[Dates are shown as month, day, year, referenced to Coordinated Universal Time (UTC). HWM, high-water mark; ft, foot. State or U.S. territory: Fla., Florida; Ga., Georgia; N.C., North Carolina; S.C., South Carolina, P.R. Puerto Rico. Datums: NAVD 88, North American Vertical Datum of 1988; NGVD 29, National Geodetic Vertical Datum of 1929; PRVD02, Puerto Rico Vertical Datum of 2002. na, not available]

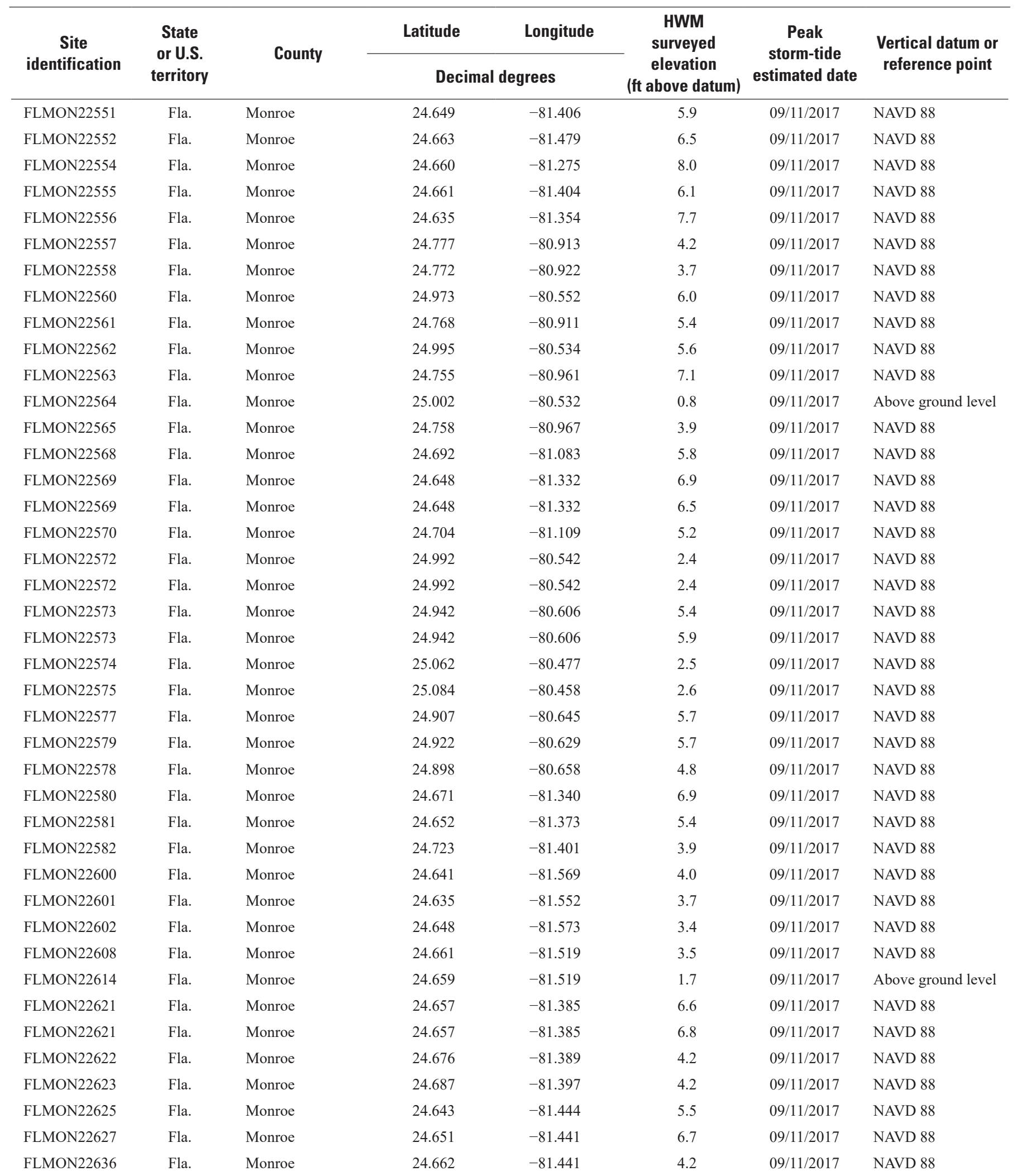


Table 5. Number of sites equipped to monitor Hurricane Irma storm tide, by State and U.S. territory.—Continued

[Dates are shown as month, day, year, referenced to Coordinated Universal Time (UTC). HWM, high-water mark; ft, foot. State or U.S. territory: Fla., Florida; Ga., Georgia; N.C., North Carolina; S.C., South Carolina, P.R. Puerto Rico. Datums: NAVD 88, North American Vertical Datum of 1988; NGVD 29, National Geodetic Vertical Datum of 1929; PRVD02, Puerto Rico Vertical Datum of 2002. na, not available]

\begin{tabular}{|c|c|c|c|c|c|c|c|}
\hline \multirow{2}{*}{$\begin{array}{c}\text { Site } \\
\text { identification }\end{array}$} & \multirow{2}{*}{$\begin{array}{c}\text { State } \\
\text { or U.S. } \\
\text { territory }\end{array}$} & \multirow{2}{*}{ County } & Latitude & Longitude & \multirow{2}{*}{$\begin{array}{c}\text { HWM } \\
\text { surveyed } \\
\text { elevation } \\
\text { (ft above datum) }\end{array}$} & \multirow{2}{*}{$\begin{array}{c}\text { Peak } \\
\text { storm-tide } \\
\text { estimated date }\end{array}$} & \multirow{2}{*}{$\begin{array}{l}\text { Vertical datum or } \\
\text { reference point }\end{array}$} \\
\hline & & & \multicolumn{2}{|c|}{ Decimal degrees } & & & \\
\hline FLMON22746 & Fla. & Monroe & 24.660 & -81.519 & 3.5 & 09/11/2017 & NAVD 88 \\
\hline FLMON22835 & Fla. & Monroe & 24.955 & -80.583 & 2.7 & 09/11/2017 & NAVD 88 \\
\hline FLMON22542 & Fla. & Monroe & 25.024 & -80.495 & 6.4 & 09/11/2017 & NAVD 88 \\
\hline FLNAS21014 & Fla. & Nassau & 30.621 & -81.439 & na & na & NAVD 88 \\
\hline FLPAS23004 & Fla. & Pasco & 28.477 & -82.194 & 65.4 & 09/11/2017 & NAVD 88 \\
\hline FLPAS23075 & Fla. & Pasco & 28.475 & -82.157 & 67.0 & 09/11/2017 & NAVD 88 \\
\hline FLPAS23080 & Fla. & Pasco & 28.475 & -82.137 & 68.6 & 09/11/2017 & NAVD 88 \\
\hline FLPAS23155 & Fla. & Pasco & 28.213 & -82.701 & 12.5 & 09/11/2017 & NAVD 88 \\
\hline FLPAS23156 & Fla. & Pasco & 28.210 & -82.701 & 10.2 & 09/11/2017 & NAVD 88 \\
\hline FLPAS23158 & Fla. & Pasco & 28.208 & -82.708 & 9.0 & 09/11/2017 & NAVD 88 \\
\hline FLPAS23150 & Fla. & Pasco & 28.218 & -82.650 & 25.5 & 09/11/2017 & NAVD 88 \\
\hline FLPAS23148 & Fla. & Pasco & 28.217 & -82.661 & 24.8 & 09/11/2017 & NAVD 88 \\
\hline FLPAS23151 & Fla. & Pasco & 28.212 & -82.674 & 21.7 & $09 / 11 / 2017$ & NAVD 88 \\
\hline FLPAS23152 & Fla. & Pasco & 28.213 & -82.679 & 20.1 & 09/11/2017 & NAVD 88 \\
\hline FLPOL23089 & Fla. & Polk & 27.748 & -81.780 & 77.7 & 09/11/2017 & NAVD 88 \\
\hline FLPOL23093 & Fla. & Polk & 27.813 & -81.794 & 86.8 & $09 / 11 / 2017$ & NAVD 88 \\
\hline FLSTJ03118 & Fla. & St. Johns & 29.949 & -81.310 & na & na & NAVD 88 \\
\hline FLSTJ03118 & Fla. & St. Johns & 29.949 & -81.310 & 6.5 & 09/11/2017 & NAVD 88 \\
\hline FLSTJ03115 & Fla. & St. Johns & 30.211 & -81.410 & na & na & NAVD 88 \\
\hline FLSTJ22830 & Fla. & St. Johns & 29.960 & -81.543 & 4.8 & $09 / 11 / 2017$ & NAVD 88 \\
\hline FLSTJ22831 & Fla. & St. Johns & 29.951 & -81.583 & 3.0 & $09 / 11 / 2017$ & NAVD 88 \\
\hline FLSTJ22834 & Fla. & St. Johns & 29.956 & -81.548 & 4.7 & 09/11/2017 & NAVD 88 \\
\hline FLSTJ22833 & Fla. & St. Johns & 29.948 & -81.575 & 3.1 & 09/11/2017 & NAVD 88 \\
\hline FLSTJ22840 & Fla. & St. Johns & 29.928 & -81.585 & 2.6 & 09/11/2017 & NAVD 88 \\
\hline FLSTJ22841 & Fla. & St. Johns & 29.967 & -81.567 & na & na & Above ground level \\
\hline FLSTJ22844 & Fla. & St. Johns & 29.924 & -81.592 & 5.0 & 09/11/2017 & NAVD 88 \\
\hline FLSTJ22847 & Fla. & St. Johns & 29.907 & -81.507 & 9.5 & $09 / 11 / 2017$ & NAVD 88 \\
\hline FLSTJ22847 & Fla. & St. Johns & 29.906 & -81.507 & 9.9 & 09/11/2017 & NAVD 88 \\
\hline FLSTJ22852 & Fla. & St. Johns & 29.910 & -81.592 & 6.3 & 09/11/2017 & NAVD 88 \\
\hline FLSTJ22856 & Fla. & St. Johns & 29.941 & -81.524 & 5.4 & 09/11/2017 & NAVD 88 \\
\hline
\end{tabular}


Table 5. Number of sites equipped to monitor Hurricane Irma storm tide, by State and U.S. territory. - Continued

[Dates are shown as month, day, year, referenced to Coordinated Universal Time (UTC). HWM, high-water mark; ft, foot. State or U.S. territory: Fla., Florida; Ga., Georgia; N.C., North Carolina; S.C., South Carolina, P.R. Puerto Rico. Datums: NAVD 88, North American Vertical Datum of 1988; NGVD 29, National Geodetic Vertical Datum of 1929; PRVD02, Puerto Rico Vertical Datum of 2002. na, not available]

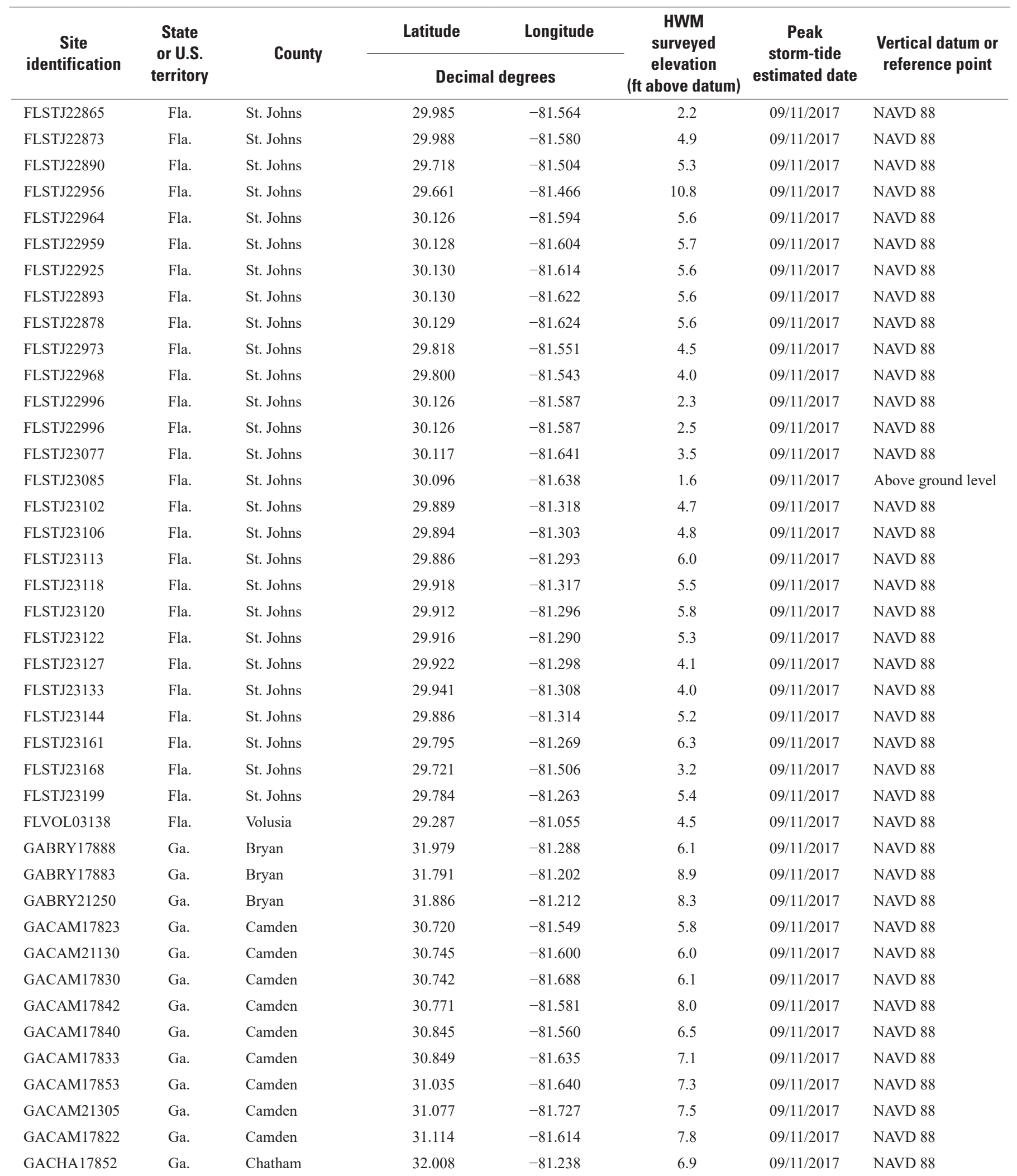


Table 5. Number of sites equipped to monitor Hurricane Irma storm tide, by State and U.S. territory.—Continued

[Dates are shown as month, day, year, referenced to Coordinated Universal Time (UTC). HWM, high-water mark; ft, foot. State or U.S. territory: Fla., Florida; Ga., Georgia; N.C., North Carolina; S.C., South Carolina, P.R. Puerto Rico. Datums: NAVD 88, North American Vertical Datum of 1988; NGVD 29, National Geodetic Vertical Datum of 1929; PRVD02, Puerto Rico Vertical Datum of 2002. na, not available]

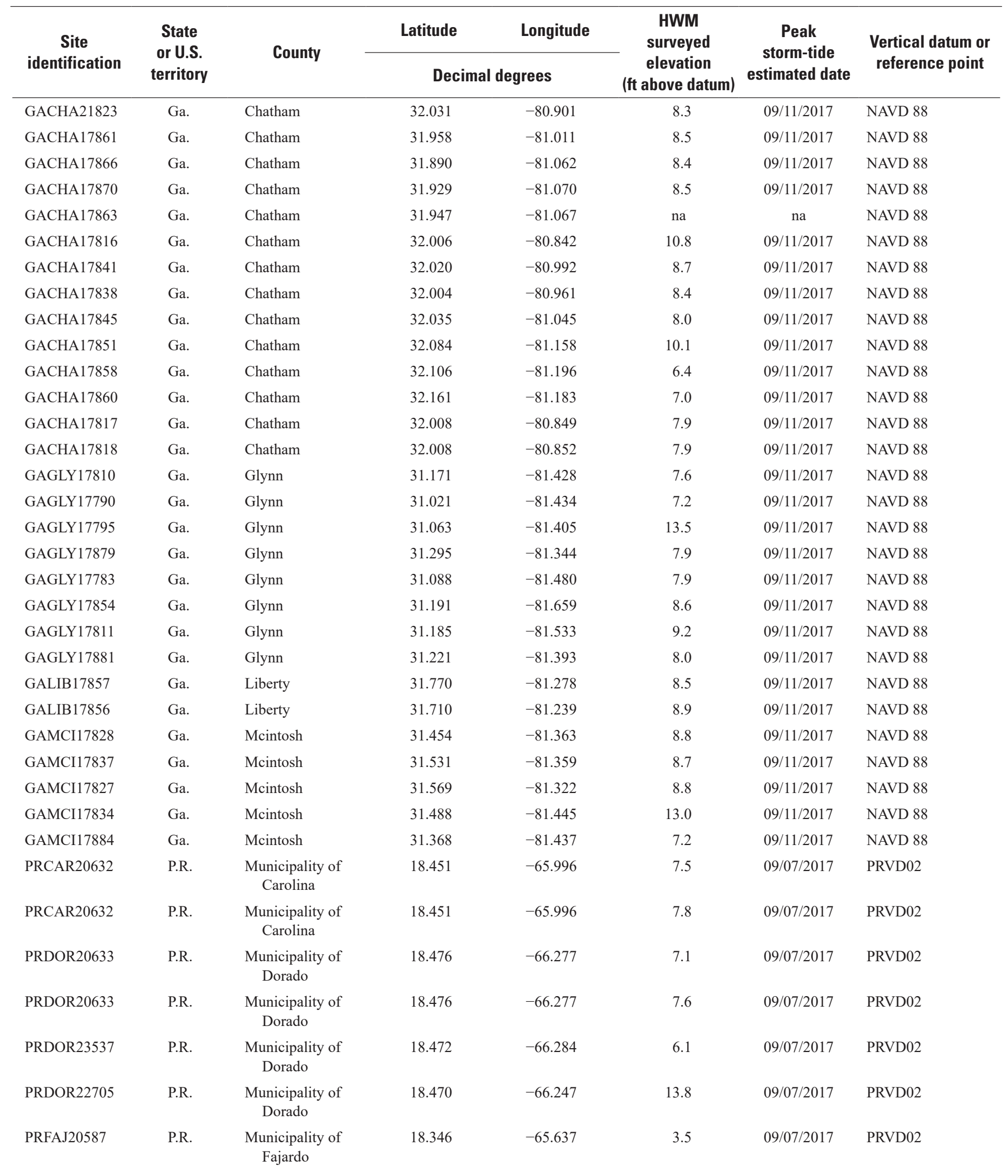


Table 5. Number of sites equipped to monitor Hurricane Irma storm tide, by State and U.S. territory.-Continued

[Dates are shown as month, day, year, referenced to Coordinated Universal Time (UTC). HWM, high-water mark; ft, foot. State or U.S. territory: Fla., Florida; Ga., Georgia; N.C., North Carolina; S.C., South Carolina, P.R. Puerto Rico. Datums: NAVD 88, North American Vertical Datum of 1988; NGVD 29, National Geodetic Vertical Datum of 1929; PRVD02, Puerto Rico Vertical Datum of 2002. na, not available]

\begin{tabular}{|c|c|c|c|c|c|c|c|}
\hline \multirow{2}{*}{$\begin{array}{c}\text { Site } \\
\text { identification }\end{array}$} & \multirow{2}{*}{$\begin{array}{l}\text { State } \\
\text { or U.S. } \\
\text { territory }\end{array}$} & \multirow{2}{*}{ County } & Latitude & Longitude & \multirow{2}{*}{$\begin{array}{c}\text { HWM } \\
\text { surveyed } \\
\text { elevation } \\
\text { (ft above datum) }\end{array}$} & \multirow{2}{*}{$\begin{array}{c}\text { Peak } \\
\text { storm-tide } \\
\text { estimated date }\end{array}$} & \multirow{2}{*}{$\begin{array}{l}\text { Vertical datum or } \\
\text { reference point }\end{array}$} \\
\hline & & & \multicolumn{2}{|c|}{ Decimal degrees } & & & \\
\hline PRFAJ20587 & P.R. & $\begin{array}{l}\text { Municipality of } \\
\text { Fajardo }\end{array}$ & 18.346 & -65.636 & 3.4 & $09 / 07 / 2017$ & PRVD02 \\
\hline PRHUM20650 & P.R. & $\begin{array}{c}\text { Municipality of } \\
\text { Humacao }\end{array}$ & 18.164 & -65.743 & 1.7 & $09 / 07 / 2017$ & PRVD02 \\
\hline PRHUM20650 & P.R. & $\begin{array}{c}\text { Municipality of } \\
\text { Humacao }\end{array}$ & 18.164 & -65.743 & 2.1 & 09/07/2017 & PRVD02 \\
\hline PRHUM20650 & P.R. & $\begin{array}{c}\text { Municipality of } \\
\text { Humacao }\end{array}$ & 18.164 & -65.743 & 2.3 & 09/07/2017 & PRVD02 \\
\hline PRLOI20636 & P.R. & $\begin{array}{l}\text { Municipality of } \\
\text { Loiza }\end{array}$ & 18.423 & -65.830 & 6.5 & 09/07/2017 & PRVD02 \\
\hline PRLOI20636 & P.R. & $\begin{array}{l}\text { Municipality of } \\
\text { Loiza }\end{array}$ & 18.423 & -65.830 & na & na & \\
\hline PRLOI22698 & P.R. & $\begin{array}{l}\text { Municipality of } \\
\text { Loiza }\end{array}$ & 18.450 & -65.904 & 5.9 & $09 / 07 / 2017$ & PRVD02 \\
\hline PRLOI20636 & P.R. & $\begin{array}{l}\text { Municipality of } \\
\text { Loiza }\end{array}$ & 18.423 & -65.830 & 7.2 & 09/07/2017 & PRVD02 \\
\hline PRTOA20649 & P.R. & $\begin{array}{l}\text { Municipality of } \\
\text { Toa Baja }\end{array}$ & 18.456 & -66.180 & 8.3 & $09 / 07 / 2017$ & PRVD02 \\
\hline PRTOA20649 & P.R. & $\begin{array}{c}\text { Municipality of } \\
\text { Toa Baja }\end{array}$ & 18.456 & -66.180 & 8.3 & $09 / 07 / 2017$ & PRVD02 \\
\hline PRTOA22708 & P.R. & $\begin{array}{l}\text { Municipality of } \\
\text { Toa Baja }\end{array}$ & 18.452 & -66.159 & 9.8 & 09/07/2017 & PRVD02 \\
\hline PRTOA23538 & P.R. & $\begin{array}{l}\text { Municipality of } \\
\text { Toa Baja }\end{array}$ & 18.453 & -66.177 & 11.4 & 09/07/2017 & PRVD02 \\
\hline PRTOA22709 & P.R. & $\begin{array}{l}\text { Municipality of } \\
\text { Toa Baja }\end{array}$ & 18.464 & -66.141 & 4.5 & $09 / 07 / 2017$ & PRVD02 \\
\hline PRVEG22702 & P.R. & $\begin{array}{l}\text { Municipality of } \\
\text { Vega Alta }\end{array}$ & 18.483 & -66.338 & 5.9 & 09/07/2017 & PRVD02 \\
\hline PRVEG23492 & P.R. & $\begin{array}{c}\text { Municipality of } \\
\text { Vega Alta }\end{array}$ & 18.489 & -66.412 & 8.3 & 09/07/2017 & PRVD02 \\
\hline PRVEG20634 & P.R. & $\begin{array}{l}\text { Municipality of } \\
\text { Vega Alta }\end{array}$ & 18.489 & -66.406 & 8.0 & $09 / 07 / 2017$ & PRVD02 \\
\hline
\end{tabular}


Table 5. Number of sites equipped to monitor Hurricane Irma storm tide, by State and U.S. territory.—Continued

[Dates are shown as month, day, year, referenced to Coordinated Universal Time (UTC). HWM, high-water mark; ft, foot. State or U.S. territory: Fla., Florida; Ga., Georgia; N.C., North Carolina; S.C., South Carolina, P.R. Puerto Rico. Datums: NAVD 88, North American Vertical Datum of 1988; NGVD 29, National Geodetic Vertical Datum of 1929; PRVD02, Puerto Rico Vertical Datum of 2002. na, not available]

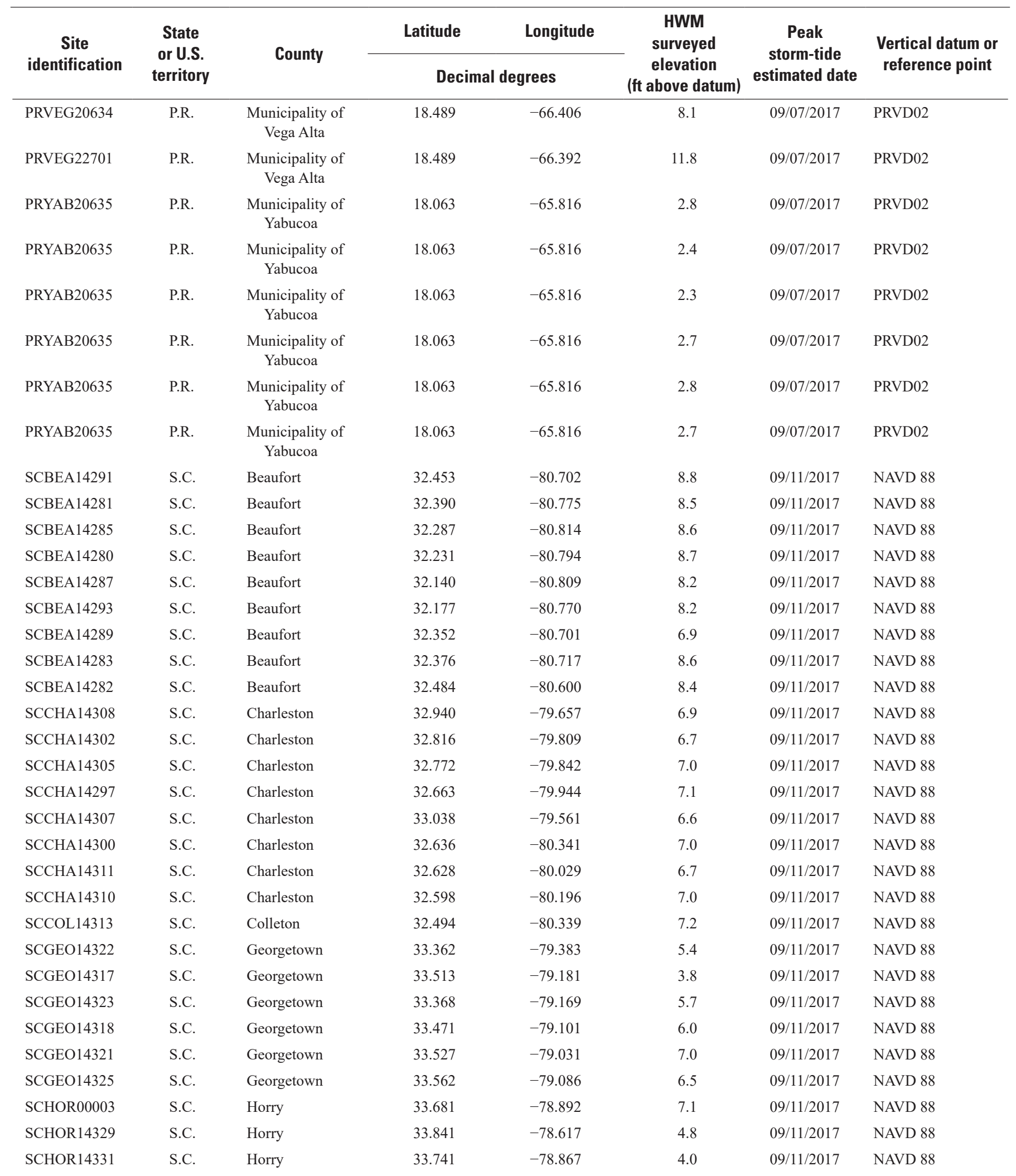


Table 5. Number of sites equipped to monitor Hurricane Irma storm tide, by State and U.S. territory.-Continued

[Dates are shown as month, day, year, referenced to Coordinated Universal Time (UTC). HWM, high-water mark; ft, foot. State or U.S. territory: Fla., Florida; Ga., Georgia; N.C., North Carolina; S.C., South Carolina, P.R. Puerto Rico. Datums: NAVD 88, North American Vertical Datum of 1988; NGVD 29, National Geodetic Vertical Datum of 1929; PRVD02, Puerto Rico Vertical Datum of 2002. na, not available]

\begin{tabular}{|c|c|c|c|c|c|c|c|}
\hline \multirow{2}{*}{$\begin{array}{c}\text { Site } \\
\text { identification }\end{array}$} & \multirow{2}{*}{$\begin{array}{c}\text { State } \\
\text { or U.S. } \\
\text { territory }\end{array}$} & \multirow{2}{*}{ County } & Latitude & Longitude & \multirow{2}{*}{$\begin{array}{c}\text { HWM } \\
\text { surveyed } \\
\text { elevation } \\
\text { (ft above datum) }\end{array}$} & \multirow{2}{*}{$\begin{array}{c}\text { Peak } \\
\text { storm-tide } \\
\text { estimated date }\end{array}$} & \multirow{2}{*}{$\begin{array}{l}\text { Vertical datum or } \\
\text { reference point }\end{array}$} \\
\hline & & & \multicolumn{2}{|c|}{ Decimal degrees } & & & \\
\hline SCHOR14328 & S.C. & Horry & 33.603 & -78.974 & 6.9 & 09/11/2017 & NAVD 88 \\
\hline SCHOR14335 & S.C. & Horry & 33.641 & -78.948 & 5.9 & 09/11/2017 & NAVD 88 \\
\hline SCHOR 17780 & S.C. & Horry & 33.759 & -78.793 & 5.4 & $09 / 11 / 2017$ & NAVD 88 \\
\hline
\end{tabular}



For more information about this publication, contact

Director, Caribbean-Florida Water Science Center

U.S. Geological Survey

4446 Pet Lane, Suite 108

Lutz, FL 33559

(813) 498-5000

For additional information visit

https://www2.usgs.gov/water/caribbeanflorida/index.html

Publishing support provided by

Lafayette Publishing Service Center 
